\title{
A HYBRID HIGH-ORDER ALGORITHM FOR RADAR CROSS SECTION COMPUTATIONS*
}

\author{
M. GANESH ${ }^{\dagger}$ AND S. C. HAWKINS $\ddagger$
}

\begin{abstract}
We describe a high-order method for computing the monostatic and bistatic radar cross section (RCS) of a class of three-dimensional targets. Our method is based on an electric field surface integral equation reformulation of the Maxwell equations. The hybrid nature of the scheme is due to approximations based on a combination of tangential and nontangential basis functions on a parametric reference spherical surface. A principal feature of the high-order algorithm is that it requires solutions of linear systems with substantially fewer unknowns than existing methods. We demonstrate that very accurate RCS values for medium (electromagnetic-) sized scatterers can be computed using a few tens of thousands of unknowns. Thus linear systems arising in the highorder method for low to medium frequency scattering can be solved using direct solves. This is extremely advantageous in monostatic RCS computations, for which transmitters and receivers are co-located and hence the discretized electromagnetic linear system must be solved for hundreds of right-hand sides corresponding to receiver locations. We demonstrate the high-order convergence of our method for several three-dimensional targets. We prove the high-order spectral accuracy of our approximations to the RCS for a class of perfect conductors described globally in spherical coordinates.
\end{abstract}

Key words. radar cross section, monostatic, bistatic, electromagnetic scattering, Maxwell's equations, surface integral, tangential basis

AMS subject classifications. 65R20, 65N35

DOI. $10.1137 / 060664859$

1. Introduction. Computer simulations of the radar cross section (RCS) of three-dimensional model targets through electromagnetic scattering are a cost effective tool for designing stealth objects. The RCS values (measured using simulated transmitters and receivers) reflect the stealth property of an obstacle [20]. Hence a prerequisite for manufacturing vehicles such as stealth aircraft is to simulate the monostatic and bistatic RCS of various geometries [30] at several frequencies to high-order accuracy. Such simulations are also useful in biological applications. For example, it is common to use light scattering measurements in laboratories to count erythrocytes (red bloods cells). Of recent interest $[19,31]$ is the simulation of the RCS of a celebrated model of an erythrocyte, which is biconcave, for an incident plane wave whose wavelength is about one tenth the diameter of the erythrocyte. Computational electromagnetic scattering algorithms with high-order accuracy facilitate efficient simulations of the RCS values.

In this work we develop, implement, and analyze a high-order algorithm to compute the monostatic and bistatic RCS of a perfectly conducting obstacle $D \subseteq \mathbb{R}^{3}$, with surface $\partial D$, situated in a homogeneous medium with vanishing conductivity, the free space permittivity $\epsilon_{0}=10^{7} /\left(4 \pi c^{2}\right) \mathrm{F} / \mathrm{m}$, and permeability $\mu_{0}=4 \pi \times 10^{-7} \mathrm{H} / \mathrm{m}$,

*Received by the editors July 12, 2006; accepted for publication (in revised form) January 5, 2007; published electronically May 29, 2007. Support of the Australian Research Council is gratefully acknowledged.

http://www.siam.org/journals/sisc/29-3/66485.html

$\dagger$ Department of Mathematical and Computer Sciences, Colorado School of Mines, Golden, CO 80401 (mganesh@mines.edu).

${ }^{\ddagger}$ School of Mathematics and Statistics, University of New South Wales, Sydney, NSW 2052, Australia (stuart@maths.unsw.edu.au). 
where $c=299792458 \mathrm{~m} / \mathrm{s}$ is the speed of light. Our simulation geometries include benchmark radar targets [30] and erythrocytes [19, 31].

The RCS of the obstacle, induced by a transmitter located in the direction $-\widehat{\boldsymbol{d}}_{0}$ and measured in decibels by a receiver located in the direction

$$
\widehat{\boldsymbol{x}}=\boldsymbol{p}(\theta, \phi)=(\sin \theta \cos \phi, \sin \theta \sin \phi, \cos \theta)^{T}, \quad \theta, \phi \in \mathbb{R},
$$

is a functional on the unit sphere (denoted by $\partial B$ ) and is defined as

$$
\sigma^{\mathrm{dB}}\left(\widehat{\boldsymbol{x}} ; \widehat{\boldsymbol{d}}_{0}\right)=10 \log _{10}\left(4 \pi\left|\boldsymbol{E}_{\infty}(\widehat{\boldsymbol{x}})\right|^{2} / k^{2}\right), \quad \widehat{\boldsymbol{x}}, \widehat{\boldsymbol{d}}_{0} \in \partial B,
$$

where

$$
\boldsymbol{E}_{\infty}(\widehat{\boldsymbol{x}})=\lim _{|\boldsymbol{x}| \rightarrow \infty} \boldsymbol{E}^{\mathrm{s}}(\boldsymbol{x}) e^{-i k|\boldsymbol{x}|}|\boldsymbol{x}|, \quad \widehat{\boldsymbol{x}}=\boldsymbol{x} /|\boldsymbol{x}| \in \partial B,
$$

is the far field pattern of the scattered field, which has frequency $\omega$ and wavenumber $k=2 \pi \omega / c$. The scattered electric field $\boldsymbol{E}^{\mathrm{s}}$ and magnetic field $\boldsymbol{H}^{\mathrm{s}}$ satisfy the time harmonic Maxwell equations

$$
\operatorname{curl} \boldsymbol{E}(\boldsymbol{x})-i k \boldsymbol{H}(\boldsymbol{x})=\mathbf{0}, \quad \operatorname{curl} \boldsymbol{H}(\boldsymbol{x})+i k \boldsymbol{E}(\boldsymbol{x})=\mathbf{0}, \quad \boldsymbol{x} \in \mathbb{R}^{3} \backslash \bar{D},
$$

and the Silver-Müller radiation condition

$$
\lim _{|\boldsymbol{x}| \rightarrow \infty}\left[\boldsymbol{H}^{\mathrm{s}}(\boldsymbol{x}) \times \boldsymbol{x}-|\boldsymbol{x}| \boldsymbol{E}^{\mathrm{s}}(\boldsymbol{x})\right]=\mathbf{0},
$$

subject to the perfect conductor boundary condition

$$
\boldsymbol{n}(\boldsymbol{x}) \times \boldsymbol{E}^{\mathrm{S}}(\boldsymbol{x})=-\boldsymbol{n}(\boldsymbol{x}) \times \boldsymbol{E}^{\mathrm{i}}(\boldsymbol{x})=: \boldsymbol{f}(\boldsymbol{x}), \quad \boldsymbol{x} \in \partial D,
$$

where $\boldsymbol{n}(\boldsymbol{x})$ denotes the unit outward normal at $\boldsymbol{x} \in \partial D$. The boundary condition in (1.5) (and hence the RCS in (1.2)) is induced by the incident plane wave $\left[\boldsymbol{E}^{\mathrm{i}}, \boldsymbol{H}^{\mathrm{i}}\right]$, with direction $\widehat{\boldsymbol{d}}_{0}$ and polarization $\widehat{\boldsymbol{p}}_{0}$ (perpendicular to $\widehat{\boldsymbol{d}}_{0}$ ), which originates from the transmitter and is defined as

$$
\boldsymbol{E}^{\mathrm{i}}(\widehat{\boldsymbol{x}})=i k \widehat{\boldsymbol{p}}_{0} e^{i k \boldsymbol{x} \cdot \widehat{\boldsymbol{d}}_{0}}, \quad \boldsymbol{H}^{\mathrm{i}}(\widehat{\boldsymbol{x}})=i k\left(\widehat{\boldsymbol{d}}_{0} \times \widehat{\boldsymbol{p}}_{0}\right) e^{i k \boldsymbol{x} \cdot \widehat{\boldsymbol{d}}_{0}} .
$$

When the transmitter and receiver are co-located, $\widehat{\boldsymbol{d}}_{0}=-\widehat{\boldsymbol{x}}$, and $\sigma^{\mathrm{dB}}$ in (1.2) is called the monostatic RCS. When the transmitter and receiver are not co-located and $\widehat{\boldsymbol{d}}_{0}$ is fixed, $\sigma^{\mathrm{dB}}$ in (1.2) is called the bistatic RCS. The RCS with receiver polarization $\widehat{\boldsymbol{p}}_{1}$ is defined by

$$
\sigma^{\mathrm{dB}}\left(\widehat{\boldsymbol{x}}, \widehat{\boldsymbol{p}}_{1} ; \widehat{\boldsymbol{d}}_{0}\right)=10 \log _{10}\left(4 \pi\left|\boldsymbol{E}_{\infty}(\widehat{\boldsymbol{x}}) \cdot \widehat{\boldsymbol{p}}_{1}\right|^{2} / k^{2}\right), \quad \widehat{\boldsymbol{x}}, \widehat{\boldsymbol{p}}_{1}, \widehat{\boldsymbol{d}}_{0} \in \partial B .
$$

The industrial standard tool for simulation of electromagnetic scattering from a perfect conductor is the Fast Illinois Solver Code (FISC); see [25, 6] and references therein for related boundary integral algorithms. FISC is based on a surface integral reformulation of the Maxwell equations and is solved using the method of moments. For medium frequency scattering problems, implementation of FISC requires solution of a dense complex linear system with hundreds of thousands of unknowns to achieve a couple of digits of accuracy. (For example, in a FISC simulation exterior to a sphere of diameter 12 times the incident wavelength, 602112 unknowns are required 
to compute the RCS with just one decimal place of accuracy [25].) Solution of large dense linear systems in FISC calculations is made possible only by using an iterative solver with matrix vector products implemented using the fast multipole method (FMM), and recently by using modified FMM algorithms based on efficient spherical harmonic expansions. A high-order algorithm for acoustic scattering was given in [4] and applied to electromagnetic scattering in [3, p. 56]. We refer the reader to $[2,3,6,9,10,19,21,23,31]$ and references therein for the current state of the art in computational electromagnetics. The existing three-dimensional electromagnetic scattering algorithms with demonstrated numerical experiments do not achieve spectral accuracy. Spectral boundary integral methods for three-dimensional model problems in acoustic, elasticity, and potential theory were considered in [12, 13, 14, 17, 29].

In [15] we considered an electromagnetic counterpart of [12] using vectorial basis functions that are tangential on the reference surface. Such vector spherical harmonics are widely used in many applications involving vector valued quantities on the sphere [11, 26, 28]. However, the approach in [15] does not yield spectrally accurate approximations [16, Remark 2]. In [16] we used a componentwise basis that is not tangential, for which we proved and demonstrated spectral accuracy. However, tangential basis functions are a natural choice for the surface integral formulation of the Maxwell equations. Further, for spherical scatterers, approximation of surface currents using tangential spherical harmonics can give almost a one third reduction in the number of unknowns required in [16]. This work is motivated by the desire to obtain this reduction by using tangential harmonics and yet obtain a spectrally accurate algorithm. We achieve the reduction and spectral accuracy in this paper with hybrid approximations, combining ideas from [15] and [16] and involving a combination of tangential and nontangential harmonics.

A clearly described parametrization of the two-dimensional manifold $\partial D$ (based on a reference domain or surface) is essential for computer implementation of any surface integral equation algorithm. We assume throughout the paper that each coordinate $(x, y, z)$ on the bounded target surface $\partial D$ can be parametrized as

$$
(x, y, z)^{T}=\left(q_{1}(\theta, \phi), q_{2}(\theta, \phi), q_{3}(\theta, \phi)\right)^{T}, \quad(x, y, z)^{T} \in \partial D, \quad \theta, \phi \in \mathbb{R},
$$

for some nonlinear functionals $q_{j}: \mathbb{R}^{2} \rightarrow \mathbb{R}$ for $j=1,2,3$ that yield, via $\boldsymbol{p}$ in (1.1), a bijective parametrization map $\boldsymbol{q}: \partial B \rightarrow \partial D$. In fact, it is sufficient to know a suitable approximation to the parametrization map $\boldsymbol{q}$, for example, based on the Fourier coefficients of $q_{j}$ for $j=1,2,3$. Our algorithm has spectral accuracy only when $\boldsymbol{q}$ is smooth.

Such a specific description of the target is required in our mathematical analysis to prove the spectral accuracy of the method. Many benchmark radar targets, such as those described in [30], satisfy the specific description (1.8) after converting the material construction (cylindrical) coordinates [30] to spherical coordinates [12], and biologically interesting models such as the erythrocyte [19,31] can be written in the parametric form (1.8). Simulation geometries considered in this work and those in [12, 16] demonstrate the wealth of obstacles satisfying (1.8). Application of the algorithm in this paper to complicated geometries is restricted by the difficulty of finding globally smooth parametrizations. Application to such geometries would require partition of the conductor surface into several charts, with appropriate use of local mappings. This approach was used in combination with a partition of unity to evaluate weakly singular integrals to spectral accuracy in [4]. Such an extension to our algorithm is nontrivial and not considered in this paper. 
In many cases the obstacle restriction imposed in this paper is not a disadvantage. For example, in forward scattering by complex structures the surface integral equation often arises through introduction of a smooth artificial interface surrounding the scatterer [1, 2, 21]. A partial differential equation form of the Maxwell equationsformulated in the interior of the artificial interface and coupled with the surface integral equation on the interface - is solved by finite elements. In inverse scattering, obtaining general qualitative information about the scatterer is more important than resolving fine details such as corners and edges.

We test our algorithm on a range of perfectly conducting scatterers. We include scatterers with concave surface features, and nonsmooth scatterers. We show that just a few tens of thousands of unknowns are sufficient in our algorithm to obtain satisfactory solutions for medium electromagnetic-sized scatterers. Such relatively small linear systems allow us to use direct solves as well as iterative solves. In the case of smooth scatterers our solutions are very accurate, with several decimal places of accuracy. In the case of nonsmooth scatterers with singularity points in their surface, our algorithm yields visually accurate solutions but does not yield the rapid convergence demonstrated for smooth scatterers. In general, only a few decimal places of global accuracy are possible for nonsmooth scatterers.

The possibility of using direct solves is a great advantage in monostatic RCS computations. For the monostatic case, since the transmitter and receiver are co-located and because hundreds of receiver measurements are required for the RCS analysis, the exterior Maxwell equations are to be solved with hundreds of boundary conditions induced by various receiver directions. This leads to the requirement of solving hundreds of linear systems with one electromagnetic discretization matrix and hundreds of different right-hand sides. A direct LU factorization of the electromagnetic matrix is very cost effective in this case. Such factorizations are not possible using surface discretization based algorithms such as FISC, because they lead to dense complex linear systems with hundreds of thousands of unknowns. We demonstrate that we can compute the monostatic RCS of medium (electromagnetic-) sized scatterers at over one thousand points in a few hours of CPU time.

The structure of the paper follows that in [16], and we recall various derivations from [16] that are essential for this paper. We follow [16] in section 2 to describe the electric field surface integral equation reformulation of (1.4)-(1.5) using a standard ansatz for electromagnetic fields exterior to the conductor surface $\partial D$, and we use (1.8) to appropriately transplant the equation onto the surface $\partial B$, the set of all unit directional vectors. In section 3 we give the details of our method based on tangential and nontangential bases for computing a spectrally accurate approximation to the surface current on $\partial D$ that solves the surface integral equation. In section 4 we describe an efficient way to use the surface current to compute the RCS of the scatterer. In section 5 we present numerical results showing the high-order convergence of our method.

Throughout the paper we denote by $\underline{C}(\partial D)$ and $\underline{C}^{0, \alpha}(\partial D)$, respectively, the spaces of all continuous and uniformly Hölder continuous vector fields on $\partial D$, where $\alpha$ is the Hölder exponent. We denote by $\underline{\mathcal{I}}(\partial D)$ and $\underline{\mathcal{T}}^{0, \alpha}(\partial D)$, respectively, the spaces of all continuous and uniformly Hölder continuous tangential vector fields on $\partial D$.

2. Reformulation of the exterior Maxwell equations. Standard boundary integral formulations for the time harmonic Maxwell equations restrict the domain of the magnetic dipole operator to $\underline{\mathcal{I}}(\partial D)[8, \mathrm{p}$. 167]. The restriction is useful because it reduces the order of the singularity of the operator. In fact, it is sufficient to 
project the density in the integral operator to $\underline{\mathcal{I}}(\partial D)$ (rather than restricting the domain) to achieve the reduction in the order of the singularity. Any vector field in $\underline{C}(\partial D)$ can be projected onto $\underline{\mathcal{I}}(\partial D)$ by applying the $3 \times 3$ matrix projection operator $\mathcal{P}(\boldsymbol{y})=I-\boldsymbol{n}(\boldsymbol{y}) \boldsymbol{n}(\boldsymbol{y})^{T}$ for $\boldsymbol{y} \in \partial D$. For $\boldsymbol{a} \in \underline{\mathcal{I}}(\partial D)$, since $\mathcal{P} \boldsymbol{a}=\boldsymbol{a}$, the generalized magnetic dipole operator

$$
(\mathcal{M} \boldsymbol{a})(\boldsymbol{x})=2 \int_{\partial D} \boldsymbol{n}(\boldsymbol{x}) \times \operatorname{curl}_{\boldsymbol{x}}\{\Phi(\boldsymbol{x}, \boldsymbol{y}) \mathcal{P}(\boldsymbol{y}) \boldsymbol{a}(\boldsymbol{y})\} d s(\boldsymbol{y}), \quad \boldsymbol{x} \in \partial D,
$$

is the same as the standard magnetic dipole operator [8, p. 167]. In (2.1),

$$
\Phi(\boldsymbol{x}, \boldsymbol{y})=\frac{1}{4 \pi} \frac{e^{i k|\boldsymbol{x}-\boldsymbol{y}|}}{|\boldsymbol{x}-\boldsymbol{y}|}
$$

is the fundamental solution of the Helmholtz equation. Application of $[8$, Theorem 6.13] shows that the linear operator $\mathcal{M}$ is bounded from $\underline{C}(\partial D)$ into $\underline{\mathcal{T}}^{0, \alpha}(\partial D)$ for $0<\alpha<1$.

Using (1.5), the boundary data $\boldsymbol{f} \in \underline{\mathcal{T}}^{0, \alpha}(\partial D)$, and so if $\boldsymbol{w} \in \underline{C}(\partial D)$ is a solution of the surface integral equation

$$
\boldsymbol{w}(\boldsymbol{x})+(\mathcal{M} \boldsymbol{w})(\boldsymbol{x})=2 \boldsymbol{f}(\boldsymbol{x}), \quad \boldsymbol{x} \in \partial D,
$$

then $\boldsymbol{w} \in \underline{\mathcal{T}}^{0, \alpha}(\partial D)$. If the wavenumber $k$ is not an interior Maxwell eigenvalue, then (2.3) has a unique solution [7, Theorem 4.23]. Throughout the paper we assume that $k$ is not an interior Maxwell eigenvalue.

Using the unique tangential solution $\boldsymbol{w}$ of (2.3), we define

$$
\boldsymbol{E}(\boldsymbol{x})=\operatorname{curl} \int_{\partial D} \Phi(\boldsymbol{x}, \boldsymbol{y}) \boldsymbol{w}(\boldsymbol{y}) d s(\boldsymbol{y}), \quad \boldsymbol{H}(\boldsymbol{x})=\frac{1}{i k} \operatorname{curl} \boldsymbol{E}(\boldsymbol{x}), \quad \boldsymbol{x} \in \mathbb{R}^{3} \backslash \bar{D}
$$

Then $\boldsymbol{E}, \boldsymbol{H}$ comprise a unique radiating solution of the harmonic Maxwell equations that satisfies the boundary condition $\boldsymbol{n}(\boldsymbol{x}) \times \boldsymbol{E}(\boldsymbol{x})=\boldsymbol{f}(\boldsymbol{x}), \boldsymbol{x} \in \partial D$ [7, Theorem 4.19]. A representation of the far field pattern required for RCS calculations can be obtained from the following asymptotics of the fundamental solution in (2.2): For $\boldsymbol{a} \in \underline{C}(\partial D)$ and as $|\boldsymbol{x}| \rightarrow \infty$ uniformly for all $\boldsymbol{y} \in \partial D$ [8, p. 164],

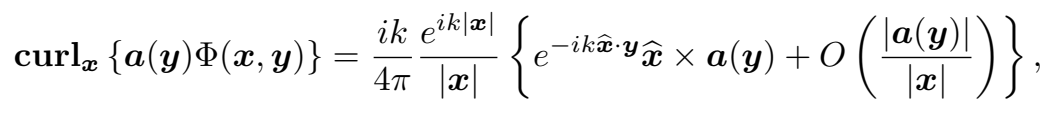

where $\widehat{\boldsymbol{x}}=\boldsymbol{x} /|\boldsymbol{x}| \in \partial B$. Using (2.4) and (2.5) in (1.3), the electric far field pattern of the scattered electric field $\boldsymbol{E}$ in (2.4) can be represented as

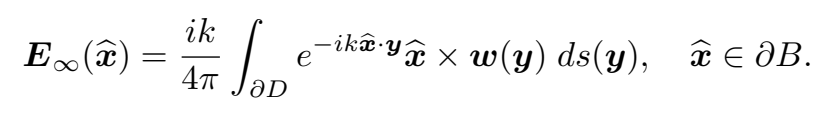

Thus high-order approximate solutions of (2.3) facilitate accurate simulation of the electromagnetic waves and measurement of the RCS using (2.6) and (1.2)-(1.7).

Using the following identity for a tangential field $\boldsymbol{b}[8$, p. 166],

$$
\begin{array}{r}
\boldsymbol{n}(\boldsymbol{x}) \times \operatorname{curl}_{\boldsymbol{x}}\{\Phi(\boldsymbol{x}, \boldsymbol{y}) \boldsymbol{b}(\boldsymbol{y})\}=\operatorname{grad}_{\boldsymbol{x}} \Phi(\boldsymbol{x}, \boldsymbol{y})[\boldsymbol{n}(\boldsymbol{x})-\boldsymbol{n}(\boldsymbol{y})]^{T} \boldsymbol{b}(\boldsymbol{y}) \\
-\boldsymbol{b}(\boldsymbol{y}) \boldsymbol{n}(\boldsymbol{x})^{T} \operatorname{grad}_{\boldsymbol{x}} \Phi(\boldsymbol{x}, \boldsymbol{y}),
\end{array}
$$


and (2.1), (2.7), and (2.2), the generalized magnetic dipole operator can be written as

$$
\mathcal{M} \boldsymbol{a}(\boldsymbol{x})=\int_{\partial D}\left[\frac{1}{|\boldsymbol{x}-\boldsymbol{y}|} m_{1}(\boldsymbol{x}, \boldsymbol{y})+m_{2}(\boldsymbol{x}, \boldsymbol{y})\right] \mathcal{P}(\boldsymbol{y}) \boldsymbol{a}(\boldsymbol{y}) d s(\boldsymbol{y}), \quad \boldsymbol{x} \in \partial D,
$$

where for $i=1,2$,

$$
\begin{aligned}
m_{i}(\boldsymbol{x}, \boldsymbol{y})=m_{i, 1}(\boldsymbol{x}, \boldsymbol{y}) \frac{1}{|\boldsymbol{x}-\boldsymbol{y}|^{2}}(\boldsymbol{x}-\boldsymbol{y})[\boldsymbol{n}(\boldsymbol{x})-\boldsymbol{n}(\boldsymbol{y})]^{T} \\
+m_{i, 2}(\boldsymbol{x}, \boldsymbol{y}) \frac{1}{|\boldsymbol{x}-\boldsymbol{y}|^{2}} \boldsymbol{n}(\boldsymbol{x})^{T}(\boldsymbol{x}-\boldsymbol{y}) I+m_{i, 3}(\boldsymbol{x}, \boldsymbol{y}),
\end{aligned}
$$

with $I$ being the $3 \times 3$ identity matrix, $m_{2,3}=0$ the $3 \times 3$ zero matrix, and

$$
\begin{aligned}
m_{1,1}(\boldsymbol{x}, \boldsymbol{y}) & =-\frac{1}{2 \pi} t(\boldsymbol{x}, \boldsymbol{y}), \quad m_{1,2}(\boldsymbol{x}, \boldsymbol{y})=\frac{1}{2 \pi} t(\boldsymbol{x}, \boldsymbol{y}), \\
m_{1,3}(\boldsymbol{x}, \boldsymbol{y}) & =\frac{1}{2 \pi} k s(\boldsymbol{x}, \boldsymbol{y})\left[\boldsymbol{n}(\boldsymbol{x})^{T}(\boldsymbol{x}-\boldsymbol{y}) I-(\boldsymbol{x}-\boldsymbol{y})(\boldsymbol{n}(\boldsymbol{x})-\boldsymbol{n}(\boldsymbol{y}))^{T}\right], \\
m_{2,1}(\boldsymbol{x}, \boldsymbol{y}) & =\frac{1}{2 \pi}[i k t(\boldsymbol{x}, \boldsymbol{y})-i s(\boldsymbol{x}, \boldsymbol{y})], \\
s(\boldsymbol{x}, \boldsymbol{y}) & =\left\{\begin{array}{ll}
\sin (k|\boldsymbol{x}-\boldsymbol{y}|) /|\boldsymbol{x}-\boldsymbol{y}| & \text { if } \boldsymbol{x} \neq \boldsymbol{y}, \\
k & \text { if } \boldsymbol{x}=\boldsymbol{y},
\end{array} \quad t(\boldsymbol{x}, \boldsymbol{y})=\cos (k|\boldsymbol{x}-\boldsymbol{y}|) .\right.
\end{aligned}
$$

Each $m_{i, j}(i=1,2, j=1,2,3)$ is infinitely differentiable on $\mathbb{R}^{3} \times \mathbb{R}^{3}$. It is important for our spectrally accurate method to separate the kernel of $\mathcal{M}$ in (2.1) into its weakly singular and smooth parts in this way. Next we use the bijective parametrization $\boldsymbol{q}: \partial B \rightarrow \partial D$ to transplant (2.8) onto the reference spherical surface, which facilitates exact treatment of the weakly singular part using a singularity division technique.

For a given density field $\boldsymbol{A} \in \underline{C}(\partial B)$, using the change of variables, we derive the equivalent form of (2.8),

$$
\mathcal{M} \boldsymbol{A}(\widehat{\boldsymbol{x}})=\mathcal{M}_{1} \boldsymbol{A}(\widehat{\boldsymbol{x}})+\mathcal{M}_{2} \boldsymbol{A}(\widehat{\boldsymbol{x}}), \quad \widehat{\boldsymbol{x}} \in \partial B,
$$

with

$$
\begin{aligned}
& \mathcal{M}_{1} \boldsymbol{A}(\widehat{\boldsymbol{x}})=\int_{\partial B} \frac{1}{|\widehat{\boldsymbol{x}}-\widehat{\boldsymbol{y}}|} M_{1}(\widehat{\boldsymbol{x}}, \widehat{\boldsymbol{y}}) \boldsymbol{A}(\widehat{\boldsymbol{y}}) d s(\widehat{\boldsymbol{y}}) \\
& \mathcal{M}_{2} \boldsymbol{A}(\widehat{\boldsymbol{x}})=\int_{\partial B} M_{2}(\widehat{\boldsymbol{x}}, \widehat{\boldsymbol{y}}) \boldsymbol{A}(\widehat{\boldsymbol{y}}) d s(\widehat{\boldsymbol{y}})
\end{aligned}
$$

where for each $\widehat{\boldsymbol{x}}, \widehat{\boldsymbol{y}} \in \partial B, M_{1}(\widehat{\boldsymbol{x}}, \widehat{\boldsymbol{y}})$ and $M_{2}(\widehat{\boldsymbol{x}}, \widehat{\boldsymbol{y}})$ are $3 \times 3$ matrices defined by

$$
\begin{aligned}
& M_{1}(\widehat{\boldsymbol{x}}, \widehat{\boldsymbol{y}})=R(\widehat{\boldsymbol{x}}, \widehat{\boldsymbol{y}}) J(\widehat{\boldsymbol{y}}) m_{1}(\boldsymbol{q}(\widehat{\boldsymbol{x}}), \boldsymbol{q}(\widehat{\boldsymbol{y}})) \mathcal{P}(\boldsymbol{q}(\widehat{\boldsymbol{y}})), \\
& M_{2}(\widehat{\boldsymbol{x}}, \widehat{\boldsymbol{y}})=J(\widehat{\boldsymbol{y}}) m_{2}(\boldsymbol{q}(\widehat{\boldsymbol{x}}), \boldsymbol{q}(\widehat{\boldsymbol{y}})) \mathcal{P}(\boldsymbol{q}(\widehat{\boldsymbol{y}})),
\end{aligned}
$$

$J$ is the Jacobian of $\boldsymbol{q}$, and

$$
R(\widehat{\boldsymbol{x}}, \widehat{\boldsymbol{y}}):=\frac{|\widehat{\boldsymbol{x}}-\widehat{\boldsymbol{y}}|}{|\boldsymbol{q}(\widehat{\boldsymbol{x}})-\boldsymbol{q}(\widehat{\boldsymbol{y}})|} .
$$

The integral operator $\mathcal{M}_{2}$ has a smooth kernel that can be approximated to spectral accuracy using spherical polynomials. Spectrally accurate approximation of 
the weakly singular operator $\mathcal{M}_{1}$ can be achieved by introducing a rotated coordinate system on $\partial B$, under which the weak singularity in the transformed operator occurs only at the north pole. To this end, we first define the $3 \times 3$ rotation matrices corresponding to positive rotations by $\psi$ about the $z$-axis and $y$-axis:

$$
\mathcal{R}_{z}(\psi):=\left(\begin{array}{ccc}
\cos \psi & -\sin \psi & 0 \\
\sin \psi & \cos \psi & 0 \\
0 & 0 & 1
\end{array}\right), \quad \mathcal{R}_{y}(\psi):=\left(\begin{array}{ccc}
\cos \psi & 0 & \sin \psi \\
0 & 1 & 0 \\
-\sin \psi & 0 & \cos \psi
\end{array}\right)
$$

For each $\widehat{\boldsymbol{x}}=\boldsymbol{p}(\theta, \phi)$, the orthogonal matrix

$$
T_{\widehat{\boldsymbol{x}}}:=\mathcal{R}_{z}(\phi) \mathcal{R}_{y}(-\theta) \mathcal{R}_{z}(-\phi)
$$

maps $\widehat{\boldsymbol{x}}$ to the north pole $\widehat{\boldsymbol{n}}=(0,0,1)^{T}$. The matrix $T_{\widehat{\boldsymbol{x}}}$ induces a linear transformation

$$
\mathcal{T}_{\widehat{\boldsymbol{x}}} \boldsymbol{A}(\widehat{\boldsymbol{z}}):=\boldsymbol{A}\left(T_{\widehat{\boldsymbol{x}}}^{-1} \widehat{\boldsymbol{z}}\right), \quad \widehat{\boldsymbol{z}} \in \partial B, \quad \boldsymbol{A} \in \underline{C}(\partial B),
$$

and its bivariate analogue

$$
\mathcal{T}_{\widehat{\boldsymbol{x}}} \boldsymbol{A}\left(\widehat{\boldsymbol{z}}_{1}, \widehat{\boldsymbol{z}}_{2}\right):=\boldsymbol{A}\left(T_{\widehat{\boldsymbol{x}}}^{-1} \widehat{\boldsymbol{z}}_{1}, T_{\widehat{\boldsymbol{x}}}^{-1} \widehat{\boldsymbol{z}}_{2}\right), \quad \widehat{\boldsymbol{z}}_{1}, \widehat{\boldsymbol{z}}_{2} \in \partial B, \quad \boldsymbol{A} \in \underline{C}(\partial B \times \partial B) .
$$

Using the orthogonality of $T_{\widehat{\boldsymbol{x}}}$, we have, for $\widehat{\boldsymbol{x}}, \widehat{\boldsymbol{z}} \in \partial B$ and $\widehat{\boldsymbol{y}}=T_{\widehat{\boldsymbol{x}}}^{-1} \widehat{\boldsymbol{z}}$,

$$
|\widehat{\boldsymbol{x}}-\widehat{\boldsymbol{y}}|=\left|T_{\widehat{\boldsymbol{x}}}^{-1}(\widehat{\boldsymbol{n}}-\widehat{\boldsymbol{z}})\right|=|\widehat{\boldsymbol{n}}-\widehat{\boldsymbol{z}}| .
$$

Using (2.12) and the fact that the surface measure on $\partial B$ is invariant,

$$
\mathcal{M}_{1} \boldsymbol{A}(\widehat{\boldsymbol{x}})=\int_{\partial B} \frac{1}{|\widehat{\boldsymbol{n}}-\widehat{\boldsymbol{z}}|} \mathcal{T}_{\widehat{\boldsymbol{x}}} M_{1}(\widehat{\boldsymbol{n}}, \widehat{\boldsymbol{z}}) \mathcal{T}_{\widehat{\boldsymbol{x}}} \boldsymbol{A}(\widehat{\boldsymbol{z}}) d s(\widehat{\boldsymbol{z}}), \quad \boldsymbol{A} \in \underline{C}(\partial B) .
$$

The transformed coordinate system has two crucial benefits. First, in spherical polar coordinates, with $\widehat{\boldsymbol{z}}=\boldsymbol{p}\left(\theta^{\prime}, \phi^{\prime}\right)$, the denominator $|\widehat{\boldsymbol{n}}-\widehat{\boldsymbol{z}}|=2 \sin \theta^{\prime} / 2$ in (2.22) is canceled out by the surface element $\sin \theta^{\prime} d \theta^{\prime} d \phi^{\prime}$. Second, the operator $\left(\theta^{\prime}, \phi^{\prime}\right) \mapsto$ $\mathcal{T}_{\widehat{\boldsymbol{x}}} M_{1}\left(\widehat{\boldsymbol{n}}, \boldsymbol{p}\left(\theta^{\prime}, \phi^{\prime}\right)\right)$ is infinitely continuously differentiable, with all derivatives $2 \pi$ periodic in each variable, and each partial derivative is uniformly bounded with respect to $\widehat{\boldsymbol{x}} \in \partial B$ (see the proof of [16, Theorem 1]).

Finally, we write $\boldsymbol{W}(\widehat{\boldsymbol{x}})=\boldsymbol{w}(\boldsymbol{q}(\widehat{\boldsymbol{x}}))$ and $\boldsymbol{F}(\widehat{\boldsymbol{x}})=\boldsymbol{f}(\boldsymbol{q}(\widehat{\boldsymbol{x}}))$, and we use (2.22) and (2.13) to derive the transplant of (2.3) onto $\partial B$,

$$
\boldsymbol{W}(\widehat{\boldsymbol{x}})+\mathcal{M} \boldsymbol{W}(\widehat{\boldsymbol{x}})=2 \boldsymbol{F}(\widehat{\boldsymbol{x}}), \quad \widehat{\boldsymbol{x}} \in \partial B, \quad \boldsymbol{W}=\boldsymbol{w} \circ \boldsymbol{q}, \quad \boldsymbol{F}=\boldsymbol{f} \circ \boldsymbol{q} .
$$

In (2.23) we have an ideal reformulation of (2.3) to obtain high-order approximation of the surface current $\boldsymbol{w}$ through a spectrally accurate evaluation of (2.11), where we approximate both the smooth kernels and the transplanted surface current in a hybrid way from the space of vector spherical polynomials.

3. A computer implementable spectral algorithm. In this section, we describe a high-order fully discrete algorithm that requires no further approximations (such as approximations of Galerkin integrals) to compute spectrally accurate approximate solutions of (2.23). Details required for computer implementation of our algorithm are in section 3.3. 
In the special case where the scatterer $\partial D$ is spherical, we seek a spectrally accurate tangential approximation to the solution of (2.23) in the tangential harmonics

$$
\underline{\mathbb{T}}_{n}=\operatorname{span}\left\{\boldsymbol{Y}_{l, j}^{(\widetilde{k})}: 1 \leq l \leq n,|j| \leq l, \widetilde{k}=1,2\right\}
$$

where

$$
\boldsymbol{Y}_{l, j}^{(1)}(\widehat{\boldsymbol{x}})=\frac{1}{\sqrt{l(l+1)}} \operatorname{Grad} Y_{l, j}(\widehat{\boldsymbol{x}}), \quad \boldsymbol{Y}_{l, j}^{(2)}(\widehat{\boldsymbol{x}})=\widehat{\boldsymbol{x}} \times \boldsymbol{Y}_{l, j}^{(1)}(\widehat{\boldsymbol{x}}), \quad \widehat{\boldsymbol{x}} \in \partial B
$$

where Grad is the surface gradient [8, p. 167] and using (1.1),

$$
Y_{l, j}(\widehat{\boldsymbol{x}})=(-1)^{(j+|j|) / 2} \sqrt{\frac{2 l+1}{4 \pi} \frac{(l-|j|) !}{(l+|j|) !}} P_{l}^{|j|}(\cos \theta) e^{i j \phi}, \quad 0 \leq l \leq n,|j| \leq l,
$$

with $P_{l}^{|j|}$ being the associated Legendre functions. The vector spherical harmonics $\boldsymbol{Y}_{l, j}^{(1)}$ and $\boldsymbol{Y}_{l, j}^{(2)}$ are tangential on $\partial B$.

In the case of a nonspherical scatterer, since the solution of the transplanted equation (2.23) need not be tangential on $\partial B$, we seek a spectrally accurate approximation in the augmented space

$$
\underline{\mathbb{Q}}_{n}=\underline{\mathbb{T}}_{n} \oplus \operatorname{span}\left\{\boldsymbol{Y}_{l, j}^{(3)}: 0 \leq l \leq n,|j| \leq l\right\}
$$

where

$$
\boldsymbol{Y}_{l, j}^{(3)}(\widehat{\boldsymbol{x}})=\widehat{\boldsymbol{x}} Y_{l, j}(\widehat{\boldsymbol{x}}), \quad \widehat{\boldsymbol{x}} \in \partial B .
$$

Using (1.1), (3.2), (3.1), and (3.4), for $\widetilde{k}=1,2,3$, we have the convenient representation

$$
\boldsymbol{Y}_{l, j}^{(\widetilde{k})}(\widehat{\boldsymbol{x}})=\sum_{t=1}^{3} \alpha_{l, j}^{(\widetilde{k}, t)}(\theta) e^{i j \phi} \boldsymbol{v}^{(t)}(\theta, \phi), \quad \widehat{\boldsymbol{x}} \in \partial B
$$

where, denoting the normalizing coefficient in (3.2) by $c_{l}^{j}$,

$$
\begin{aligned}
\boldsymbol{v}^{(1)}(\theta, \phi) & =(\cos \theta \cos \phi, \cos \theta \sin \phi,-\sin \theta)^{T}, \boldsymbol{v}^{(2)}(\theta, \phi)=(-\sin \phi, \cos \phi, 0)^{T}, \\
\boldsymbol{v}^{(3)}(\theta, \phi) & =(\sin \theta \cos \phi, \sin \theta \sin \phi, \cos \theta)^{T} \\
\alpha_{l, j}^{(1,1)}(\theta) & =\alpha_{l, j}^{(2,2)}(\theta)=c_{l}^{j} P_{l}^{|j|^{\prime}}(\cos \theta) / \sqrt{l(l+1)} \\
\alpha_{l, j}^{(1,2)}(\theta) & =-\alpha_{l, j}^{(2,1)}(\theta)=c_{l}^{j}(i j / \sin \theta) P_{l}^{|j|}(\cos \theta) / \sqrt{l(l+1)}, \\
\alpha_{l, j}^{(3,3)}(\theta) & =c_{l}^{j} P_{l}^{|j|}(\cos \theta) \\
\alpha_{l, j}^{(1,3)}(\theta) & =\alpha_{l, j}^{(2,3)}(\theta)=\alpha_{l, j}^{(3,1)}(\theta)=\alpha_{l, j}^{(3,2)}(\theta)=0 .
\end{aligned}
$$

The standard Galerkin scheme for (2.23) requires evaluation of the $L_{2}$ inner product integrals

$$
(\boldsymbol{G}, \boldsymbol{H})=\mathcal{I}\left(\overline{\boldsymbol{H}}^{T} \boldsymbol{G}\right)=\int_{\partial B} \overline{\boldsymbol{H}(\widehat{\boldsymbol{x}})}^{T} \boldsymbol{G}(\widehat{\boldsymbol{x}}) d s(\widehat{\boldsymbol{x}}), \quad \boldsymbol{G}, \boldsymbol{H} \in \underline{C}(\partial B) .
$$


In practice, a quadrature rule on $\partial B$ is required to evaluate the integrals in (3.6). We approximate the inner product integrals using the Gauss-rectangle quadrature rule

$$
\int_{\partial B} G(\widehat{\boldsymbol{x}}) d s(\widehat{\boldsymbol{x}}) \approx Q_{N}(G)=\sum_{r=0}^{2 N+1} \sum_{s=1}^{N+1} \mu_{r}^{N} \nu_{s}^{N} G\left(\widehat{\boldsymbol{z}}_{r s}^{N}\right), \quad G \in C(\partial B),
$$

where, using (1.1), $\widehat{\boldsymbol{z}}_{r s}^{N}=\boldsymbol{p}\left(\theta_{s}^{N}, \phi_{r}^{N}\right), \theta_{s}^{N}=\cos ^{-1} z_{s}, z_{s}$ are the zeros of the Legendre polynomial of degree $N+1, \nu_{s}^{N}$ are the corresponding Gauss-Legendre weights $(s=$ $1, \ldots, N+1)$, and

$$
\mu_{r}^{N}=\frac{\pi}{N+1}, \quad \phi_{r}^{N}=\frac{r \pi}{N+1}, \quad r=0, \ldots, 2 N+1 .
$$

Since the quadrature (3.7) is exact for spherical polynomials of degree at most $2 N$, it induces the discrete inner product

$$
(\boldsymbol{G}, \boldsymbol{H})_{N}=Q_{N}\left(\overline{\boldsymbol{H}}^{T} \boldsymbol{G}\right)=\sum_{r=0}^{2 N+1} \sum_{s=1}^{N+1} \mu_{r}^{N} \nu_{s}^{N}{\overline{\boldsymbol{H}\left(\widehat{\boldsymbol{z}}_{r s}^{N}\right)}}^{T} \boldsymbol{G}\left(\widehat{\boldsymbol{z}}_{r s}^{N}\right), \quad \boldsymbol{G}, \boldsymbol{H} \in \underline{C}(\partial B),
$$

on $\mathbb{P}_{N}$, the space of vector spherical polynomials whose Euclidean components are spherical polynomials of degree not greater than $N$. That is, $(\boldsymbol{G}, \boldsymbol{H})=(\boldsymbol{G}, \boldsymbol{H})_{N}$ for $\boldsymbol{G}, \boldsymbol{H} \in \underline{\mathbb{P}}_{N}$. Since $\boldsymbol{Y}_{n, j}^{(\widetilde{k})}$ for $\widetilde{k}=1,2,3$ in (3.1)-(3.4) is a vector spherical polynomial of degree $n+1$, we have $\underline{\mathbb{Q}}_{n} \subseteq \underline{\mathbb{P}}_{n+1}$, and hence the vector spherical harmonics in (3.1) and (3.4) are orthonormal with respect to the discrete inner product (3.9) with $N=n+1$.

We approximate the transplanted surface current in (2.23) by a computable projection onto the finite dimensional space $\mathbb{Q}_{n}$. In particular, we approximate the theoretical solution $\boldsymbol{W}$ of (2.23) using a computable representation $\underline{\mathcal{O}}_{n} \boldsymbol{W}$, where $\underline{\mathcal{O}}_{n}$ is a fully discrete projection operator defined as

$$
\underline{\mathcal{O}}_{n} \boldsymbol{A}=\sum_{l=0}^{n} \sum_{|j| \leq l} \sum_{\widetilde{k}=1}^{3}\left(\boldsymbol{A}, \boldsymbol{Y}_{l, j}^{(\widetilde{k})}\right)_{n+1} \boldsymbol{Y}_{l, j}^{(\widetilde{k})}, \quad \boldsymbol{A} \in \underline{C}(\partial B)
$$

where, for convenience, throughout the paper, we use the notation $\boldsymbol{Y}_{00}^{(1)}=\boldsymbol{Y}_{00}^{(2)}=\mathbf{0}$. Using the orthonormality of the vector harmonics with respect to the discrete inner product in (3.10), we get $\underline{\mathcal{O}}_{n} \boldsymbol{Y}_{l, j}^{(\widetilde{k})}=\boldsymbol{Y}_{l, j}^{(\widetilde{k})}$ for $0 \leq l \leq n,|j| \leq l, 1 \leq \widetilde{k} \leq 3$. Throughout the paper, when $\partial D$ is a sphere, we replace $\sum_{\widetilde{k}=1}^{3}$ in (3.10) by $\sum_{\widetilde{k}=1}^{2}$, and $\underline{\mathbb{Q}}_{n}$ by $\underline{\mathbb{T}}_{n}$.

3.1. Fully discrete approximations of magnetic dipole integrals. For evaluation of inner integrals in the Galerkin scheme, for example, $\mathcal{M}_{1} \boldsymbol{A}(\widehat{\boldsymbol{x}})$, we need to approximate the integrand $\mathcal{T}_{\widehat{\boldsymbol{x}}} M_{1}(\widehat{\boldsymbol{n}}, \cdot) \mathcal{T}_{\widehat{\boldsymbol{x}}} \boldsymbol{A}(\cdot)$ in $(2.22)$ for $\boldsymbol{A} \in \underline{C}(\partial B), \widehat{\boldsymbol{x}} \in \partial B$. One approach is to project this integrand using the fully discrete operator $\underline{\mathcal{O}}_{n}$ in (3.10), as in [15]. However, as described in [16, Remark 2], using a technical analysis, such an approximation, leads to stagnated convergence instead of the spectral accuracy we seek in this work. In this hybrid scheme, to approximate the inner integral, we use the componentwise nontangential harmonics defined using (3.2) as

$$
\boldsymbol{Y}_{l, j, \widetilde{k}}=Y_{l, j} \boldsymbol{e}_{\widetilde{k}}, \quad 0 \leq l \leq n^{\prime},|j| \leq l, 1 \leq \widetilde{k} \leq 3,
$$


where $\boldsymbol{e}_{\widetilde{k}}$ denotes the $\widetilde{k}$ th Euclidean vector. To this end, analogous to (3.10), using the discrete inner product (3.9), we define a fully discrete orthogonal projection operator

$$
\underline{\mathcal{L}}_{n^{\prime}} \boldsymbol{A}=\sum_{l=0}^{n^{\prime}} \sum_{|j| \leq l} \sum_{\widetilde{k}=1}^{3}\left(\boldsymbol{A}, \boldsymbol{Y}_{l, j, \tilde{k}}\right)_{n^{\prime}} \boldsymbol{Y}_{l, j, \tilde{k}}, \quad \boldsymbol{A} \in \underline{C}(\partial B) .
$$

Clearly $\underline{\mathcal{L}}_{n^{\prime}} \boldsymbol{Y}_{l, j, \widetilde{k}}=\boldsymbol{Y}_{l, j, \widetilde{k}}$ for $0 \leq l \leq n^{\prime},|j| \leq l, 1 \leq \widetilde{k} \leq 3$. Here, $n^{\prime}$ depends on $n$ and will be specified later in this section. We approximate $\mathcal{T}_{\widehat{\boldsymbol{x}}} M_{1}(\widehat{\boldsymbol{n}}, \cdot) \mathcal{T}_{\widehat{\boldsymbol{x}}} \boldsymbol{A}(\cdot)$ in (2.22) by $\underline{\mathcal{L}}_{n^{\prime}}\left\{\mathcal{T}_{\widehat{\boldsymbol{x}}} M_{1}(\widehat{\boldsymbol{n}}, \cdot) \mathcal{T}_{\widehat{\boldsymbol{x}}} \boldsymbol{A}(\cdot)\right\}$ and follow the details in [16].

More precisely, for $\boldsymbol{A} \in \underline{C}(\partial B)$ and $\widehat{\boldsymbol{x}} \in \partial B$, using (2.22) and (3.12), the approximation to $\mathcal{M}_{1} \boldsymbol{A}(\widehat{\boldsymbol{x}})$ is

$$
\begin{aligned}
\mathcal{M}_{1, n^{\prime}} \boldsymbol{A}(\widehat{\boldsymbol{x}}) & =\int_{\partial B} \frac{1}{|\widehat{\boldsymbol{n}}-\widehat{\boldsymbol{z}}|} \underline{\mathcal{L}}_{n^{\prime}}\left\{\mathcal{T}_{\widehat{\boldsymbol{x}}} M_{1}(\widehat{\boldsymbol{n}}, \cdot) \mathcal{T}_{\widehat{\boldsymbol{x}}} \boldsymbol{A}(\cdot)\right\}(\widehat{\boldsymbol{z}}) d s(\widehat{\boldsymbol{z}}) \\
(3.13) & =\int_{\partial B} \frac{1}{|\widehat{\boldsymbol{n}}-\widehat{\boldsymbol{z}}|} \sum_{l=0}^{n^{\prime}} \sum_{|j| \leq l} \sum_{\widetilde{k}=1}^{3}\left(\mathcal{T}_{\widehat{\boldsymbol{x}}} M_{1}(\widehat{\boldsymbol{n}}, \cdot) \mathcal{T}_{\widehat{\boldsymbol{x}}} \boldsymbol{A}(\cdot), \boldsymbol{Y}_{l, j, \widetilde{k}}(\cdot)\right)_{n^{\prime}} \boldsymbol{Y}_{l, j, \widetilde{k}}(\widehat{\boldsymbol{z}}) d s(\widehat{\boldsymbol{z}}) \\
& =\sum_{l=0}^{n^{\prime}} \sum_{|j| \leq l} \sum_{\widetilde{k}=1}^{3}\left(\mathcal{T}_{\widehat{\boldsymbol{x}}} M_{1}(\widehat{\boldsymbol{n}}, \cdot) \mathcal{T}_{\widehat{\boldsymbol{x}}} \boldsymbol{A}(\cdot), \boldsymbol{Y}_{l, j, \widetilde{k}}(\cdot)\right)_{n^{\prime}} \int_{\partial B} \frac{1}{|\widehat{\boldsymbol{n}}-\widehat{\boldsymbol{z}}|} \boldsymbol{Y}_{l, j, \widetilde{k}}(\widehat{\boldsymbol{z}}) d s(\widehat{\boldsymbol{z}}) \\
& =\sum_{l=0}^{n^{\prime}} \sum_{|j| \leq l} \sum_{\widetilde{k}=1}^{3}\left(\mathcal{T}_{\widehat{\boldsymbol{x}}} M_{1}(\widehat{\boldsymbol{n}}, \cdot) \mathcal{T}_{\widehat{\boldsymbol{x}}} \boldsymbol{A}(\cdot), \boldsymbol{Y}_{l, j, \widetilde{k}}(\cdot)\right)_{n^{\prime}} \frac{4 \pi}{2 l+1} \boldsymbol{Y}_{l, j, \widetilde{k}}(\widehat{\boldsymbol{n}}) .
\end{aligned}
$$

In the last step we have used the standard eigenfunction property of the spherical harmonics [11]. Using (3.9), we expand the discrete inner product and obtain

$$
\begin{aligned}
& \mathcal{M}_{1, n^{\prime}} \boldsymbol{A}(\widehat{\boldsymbol{x}}) \\
& \left.=\sum_{l=0}^{n^{\prime}} \sum_{|j| \leq l} \sum_{\widetilde{k}=1}^{3} \sum_{s^{\prime}=1}^{n^{\prime}+1} \sum_{r^{\prime}=0}^{2 n^{\prime}+1} \mu_{r^{\prime}}^{n^{\prime}} \nu_{s^{\prime}}^{n^{\prime}} \frac{4 \pi}{2 l+1} \overline{\boldsymbol{Y}_{l, j, \widetilde{k}}\left(\widehat{\boldsymbol{z}}_{r^{\prime} s^{\prime}}^{n^{\prime}}\right.}\right)^{T} \mathcal{T}_{\widehat{\boldsymbol{x}}} M_{1}\left(\widehat{\boldsymbol{n}}, \widehat{\boldsymbol{z}}_{r^{\prime} s^{\prime}}^{n^{\prime}}\right) \mathcal{T}_{\widehat{\boldsymbol{x}}} \boldsymbol{A}\left(\widehat{\boldsymbol{z}}_{r^{\prime} s^{\prime}}^{n^{\prime}}\right) \boldsymbol{Y}_{l, j, \widetilde{k}}(\widehat{\boldsymbol{n}}) \\
& =\sum_{l=0}^{n^{\prime}} \sum_{\widetilde{k}=1}^{3} \sum_{s^{\prime}=1}^{n^{\prime}+1} \sum_{r^{\prime}=0}^{2 n^{\prime}+1} \mu_{r^{\prime}}^{n^{\prime}} \nu_{s^{\prime}}^{n^{\prime}} \frac{4 \pi}{2 l+1} \boldsymbol{e}_{\widetilde{k}} \boldsymbol{e}_{\widetilde{k}}^{T} \mathcal{T}_{\widehat{\boldsymbol{x}}} M_{1}\left(\widehat{\boldsymbol{n}}, \widehat{\boldsymbol{z}}_{r^{\prime} s^{\prime}}^{n^{\prime}}\right) \mathcal{T}_{\widehat{\boldsymbol{x}}} \boldsymbol{A}\left(\widehat{\boldsymbol{z}}_{r^{\prime} s^{\prime}}^{n^{\prime}}\right) \sum_{|j| \leq l} \overline{Y_{l, j}\left(\widehat{\boldsymbol{z}}_{r^{\prime} s^{\prime}}^{n^{\prime}}\right)} Y_{l, j}(\widehat{\boldsymbol{n}}) .
\end{aligned}
$$

Using the addition theorem for the spherical harmonics [8, Theorem 2.8], this simplifies to

$$
\begin{aligned}
\mathcal{M}_{1, n^{\prime}} \boldsymbol{A}(\widehat{\boldsymbol{x}}) & =\sum_{l=0}^{n^{\prime}} \sum_{\widetilde{k}=1}^{3} \sum_{s^{\prime}=1}^{n^{\prime}+1} \sum_{r^{\prime}=0}^{2 n^{\prime}+1} \mu_{r^{\prime}}^{n^{\prime}} \nu_{s^{\prime}}^{n^{\prime}} \boldsymbol{e}_{\widetilde{k}} \boldsymbol{e}_{\widetilde{k}}^{T} \mathcal{T}_{\widehat{\boldsymbol{x}}} M_{1}\left(\widehat{\boldsymbol{n}}, \widehat{\boldsymbol{z}}_{r^{\prime} s^{\prime}}^{n^{\prime}}\right) \mathcal{T}_{\widehat{\boldsymbol{x}}} \boldsymbol{A}\left(\widehat{\boldsymbol{z}}_{r^{\prime} s^{\prime}}^{n^{\prime}}\right) P_{l}\left(\widehat{\boldsymbol{n}} \cdot \widehat{\boldsymbol{z}}_{r^{\prime} s^{\prime}}^{n^{\prime}}\right) \\
& =\sum_{s^{\prime}=1}^{n^{\prime}+1} \sum_{r^{\prime}=0}^{2 n^{\prime}+1} \mu_{r^{\prime}}^{n^{\prime}} \nu_{s^{\prime}}^{n^{\prime}} \alpha_{s^{\prime}}^{n^{\prime}} \mathcal{T}_{\widehat{\boldsymbol{x}}} M_{1}\left(\widehat{\boldsymbol{n}}, \widehat{\boldsymbol{z}}_{r^{\prime} s^{\prime}}^{n^{\prime}}\right) \mathcal{T}_{\widehat{\boldsymbol{x}}} \boldsymbol{A}\left(\widehat{\boldsymbol{z}}_{r^{\prime} s^{\prime}}^{n^{\prime}}\right),
\end{aligned}
$$

where $\alpha_{s^{\prime}}^{n^{\prime}}=\sum_{l=0}^{n^{\prime}} P_{l}\left(\cos \theta_{s^{\prime}}^{n^{\prime}}\right)$, and we have used $\widehat{\boldsymbol{n}} \cdot \widehat{\boldsymbol{z}}_{r^{\prime} s^{\prime}}^{n^{\prime}}=\cos \theta_{s^{\prime}}^{n^{\prime}}$. Thus $\mathcal{M}_{1, n^{\prime}} \boldsymbol{A}$ is a fully discrete computable approximation to the transplanted weakly singular part of the generalized magnetic dipole operator in (2.8). 
We use a similar approximation for the smooth part of the operator. We write (2.13) in the rotated coordinate system as

$$
\mathcal{M}_{2} \boldsymbol{A}(\widehat{\boldsymbol{x}})=\int_{\partial B} \mathcal{T}_{\widehat{\boldsymbol{x}}} M_{2}(\widehat{\boldsymbol{n}}, \widehat{\boldsymbol{z}}) \mathcal{T}_{\widehat{\boldsymbol{x}}} \boldsymbol{A}(\widehat{\boldsymbol{z}}) d s(\widehat{\boldsymbol{z}}) .
$$

Our approximation to $\mathcal{M}_{2} \boldsymbol{A}$ is then

$$
\begin{aligned}
\mathcal{M}_{2, n^{\prime}} \boldsymbol{A}(\widehat{\boldsymbol{x}}) & =\int_{\partial B} \underline{\mathcal{L}}_{n^{\prime}}\left\{\mathcal{T}_{\widehat{\boldsymbol{x}}} M_{2}(\widehat{\boldsymbol{n}}, \cdot) \mathcal{T}_{\widehat{\boldsymbol{x}}} \boldsymbol{A}(\cdot)\right\}(\widehat{\boldsymbol{z}}) d s(\widehat{\boldsymbol{z}}) \\
& =\int_{\partial B} \sum_{l=0}^{n^{\prime}} \sum_{|j| \leq l} \sum_{\widetilde{k}=1}^{3}\left(\mathcal{T}_{\widehat{\boldsymbol{x}}} M_{2}(\widehat{\boldsymbol{n}}, \cdot) \mathcal{T}_{\widehat{\boldsymbol{x}}} \boldsymbol{A}(\cdot), \boldsymbol{Y}_{l, j, \widetilde{k}}(\cdot)\right)_{n^{\prime}} \boldsymbol{Y}_{l, j, \widetilde{k}}(\widehat{\boldsymbol{z}}) d s(\widehat{\boldsymbol{z}}) \\
& =\sum_{l=0}^{n^{\prime}} \sum_{|j| \leq l} \sum_{\widetilde{k}=1}^{3}\left(\mathcal{T}_{\widehat{\boldsymbol{x}}} M_{2}(\widehat{\boldsymbol{n}}, \cdot) \mathcal{T}_{\widehat{\boldsymbol{x}}} \boldsymbol{A}(\cdot), \boldsymbol{Y}_{l, j, \widetilde{k}}(\cdot)\right)_{n^{\prime}} \int_{\partial B} \boldsymbol{Y}_{l, j, \widetilde{k}}(\widehat{\boldsymbol{z}}) d s(\widehat{\boldsymbol{z}}) .
\end{aligned}
$$

Noting that $\boldsymbol{Y}_{0,0, \widetilde{k}}=\boldsymbol{e}_{\widetilde{k}} / \sqrt{4 \pi}$, the orthogonality of the $\boldsymbol{Y}_{l, j, \widetilde{k}}$ gives

$$
\mathcal{M}_{2, n^{\prime}} \boldsymbol{A}(\widehat{\boldsymbol{x}})=\sum_{\widetilde{k}=1}^{3}\left(\mathcal{T}_{\widehat{\boldsymbol{x}}} M_{2}(\widehat{\boldsymbol{n}}, \cdot) \mathcal{T}_{\widehat{\boldsymbol{x}}} \boldsymbol{A}(\cdot), \boldsymbol{e}_{\widetilde{k}}\right)_{n^{\prime}} \boldsymbol{e}_{\widetilde{k}} .
$$

Expanding the discrete inner product using (3.9),

$$
\begin{aligned}
\mathcal{M}_{2, n^{\prime}} \boldsymbol{A}(\widehat{\boldsymbol{x}}) & =\sum_{\widetilde{k}=1}^{3} \sum_{s^{\prime}=1}^{n^{\prime}+1} \sum_{r^{\prime}=0}^{2 n^{\prime}+1} \mu_{r^{\prime}}^{n^{\prime}} \nu_{s^{\prime}}^{n^{\prime}} \boldsymbol{e}_{\widetilde{k}} \boldsymbol{e}_{\widetilde{k}}^{T} \mathcal{T}_{\widehat{\boldsymbol{x}}} M_{2}\left(\widehat{\boldsymbol{n}}, \widehat{\boldsymbol{z}}_{r^{\prime} s^{\prime}}^{n^{\prime}}\right) \mathcal{T}_{\widehat{\boldsymbol{x}}} \boldsymbol{A}\left(\widehat{\boldsymbol{z}}_{r^{\prime} s^{\prime}}^{n^{\prime}}\right) \\
& =\sum_{s^{\prime}=1}^{n^{\prime}+1} \sum_{r^{\prime}=0}^{2 n^{\prime}+1} \mu_{r^{\prime}}^{n^{\prime}} \nu_{s^{\prime}}^{n^{\prime}} \mathcal{T}_{\widehat{\boldsymbol{x}}} M_{2}\left(\widehat{\boldsymbol{n}}, \widehat{\boldsymbol{z}}_{r^{\prime} s^{\prime}}^{n^{\prime}}\right) \mathcal{T}_{\widehat{\boldsymbol{x}}} \boldsymbol{A}\left(\widehat{\boldsymbol{z}}_{r^{\prime} s^{\prime}}^{n^{\prime}}\right)
\end{aligned}
$$

Combining (3.14) and (3.17), we approximate the reformulated and projected magnetic dipole operator (2.11) by the computable representation

$$
\mathcal{M}_{n^{\prime}} \boldsymbol{A}(\widehat{\boldsymbol{x}})=\mathcal{M}_{1, n^{\prime}} \boldsymbol{A}(\widehat{\boldsymbol{x}})+\mathcal{M}_{2, n^{\prime}} \boldsymbol{A}(\widehat{\boldsymbol{x}}) .
$$

Finally, we must choose $n^{\prime}$ to ensure that $\mathcal{M}_{n^{\prime}} \boldsymbol{A}$ converges to $\mathcal{M} \boldsymbol{A}$ with spectral accuracy. Following the proof of [16, Theorem 1] it can be shown that if $n^{\prime}=a n+1$ for some fixed constant $a>1$, and $n^{\prime}-n \geq 3$, then for any $s \in \mathbb{N}$, there exists $c_{s}>0$, independent of $n$ and $n^{\prime}$, such that

$$
\left\|\left(\mathcal{M}-\mathcal{M}_{n^{\prime}}\right) \boldsymbol{P}_{n}\right\|_{\infty, \partial B} \leq c_{s} \frac{1}{n^{s}}\left\|\boldsymbol{P}_{n}\right\|_{\infty, \partial B} \quad \text { for all } \boldsymbol{P}_{n} \in \underline{\mathbb{P}}_{n+1} .
$$

As in [16, Theorem 1], if the conductor surface is smooth then (3.19) holds for any number $s$. We remark that in our computational algorithm, the density function of the fully discrete magnetic dipole operator is a spherical polynomial of degree at most $n+1$. For computations in section 5 we set $n^{\prime}=2 n+1$.

3.2. Complete algorithm. We are now ready to describe the complete algorithm to solve (2.23), using the various spectrally accurate approximations described 
above. Our fully discrete scheme for $(2.23)$ is as follows: compute $\boldsymbol{W}_{n} \in \underline{\mathbb{Q}}_{n}$ such that

$$
\boldsymbol{W}_{n}+\underline{\mathcal{O}}_{n} \mathcal{M}_{n^{\prime}} \boldsymbol{W}_{n}=2 \underline{\mathcal{O}}_{n} \boldsymbol{f} .
$$

Since $\boldsymbol{W}_{n} \in \underline{\mathbb{Q}}_{n}$ can be written as

$$
\boldsymbol{W}_{n}(\widehat{\boldsymbol{x}})=\mathrm{w}_{003} \boldsymbol{Y}_{00}^{(3)}(\widehat{\boldsymbol{x}})+\sum_{l=1}^{n} \sum_{|j| \leq l} \sum_{\widetilde{k}=1}^{3} \mathrm{w}_{l j \tilde{k}} \boldsymbol{Y}_{l, j}^{(\widetilde{k})}(\widehat{\boldsymbol{x}}), \quad \widehat{\boldsymbol{x}} \in \partial B
$$

the operator equation (3.20) is equivalent to the requirement that the coefficients $\mathrm{w}_{l j \tilde{k}}$ solve the fully discrete Galerkin system

$$
\left(\boldsymbol{W}_{n}, \boldsymbol{Y}_{l^{\prime}, j^{\prime}}^{\left(k^{\prime}\right)}\right)_{n+1}+\left(\mathcal{M}_{n^{\prime}} \boldsymbol{W}_{n}, \boldsymbol{Y}_{l^{\prime}, j^{\prime}}^{\left(k^{\prime}\right)}\right)_{n+1}=2\left(\boldsymbol{F}, \boldsymbol{Y}_{l^{\prime}, j^{\prime}}^{\left(k^{\prime}\right)}\right)_{n+1}, \quad\left(l^{\prime}, j^{\prime}, k^{\prime}\right) \in \mathcal{I},
$$

where $\mathcal{I}=\{(0,0,3)\} \cup\{(\widehat{l}, \widehat{j}, \widehat{k}): 1 \leq \widehat{l} \leq n,|\widehat{j}| \leq \widehat{l}, \widehat{k}=1,2,3\}$. In matrix form, $\mathrm{w}=\left(\mathrm{w}_{l j \widetilde{k}}\right)$ satisfies

$$
(\mathrm{I}+\mathrm{M}) \mathrm{w}=2 \mathrm{f},
$$

and for $(l, j, \widetilde{k}),\left(l^{\prime}, j^{\prime}, k^{\prime}\right) \in \mathcal{I}$,

$$
\begin{aligned}
\mathrm{M}_{l^{\prime} j^{\prime} k^{\prime}, l j \widetilde{k}} & =\left(\mathcal{M}_{n^{\prime}} \boldsymbol{Y}_{l, j}^{(\widetilde{k})}, \boldsymbol{Y}_{l^{\prime}, j^{\prime}}^{\left(k^{\prime}\right)}\right)_{n+1}, \quad \mathrm{I}_{l^{\prime} j^{\prime} k^{\prime}, l j \widetilde{k}}=\delta_{l l^{\prime}} \delta_{j j^{\prime}} \delta_{\widetilde{k} k^{\prime}}, \\
\mathrm{f}_{l^{\prime} j^{\prime} k^{\prime}} & =\left(\boldsymbol{F}, \boldsymbol{Y}_{l^{\prime}, j^{\prime}}^{\left(k^{\prime}\right)}\right)_{n+1} .
\end{aligned}
$$

Our corresponding approximation to the solution $\boldsymbol{w}$ of (2.3) is then

$$
\boldsymbol{w}_{n}(\boldsymbol{x})=\boldsymbol{W}_{n}\left(\boldsymbol{q}^{-1}(\boldsymbol{x})\right), \quad \boldsymbol{x} \in \partial D .
$$

Using (3.19) and following the proof of [16, Theorem 3] (with $\underline{\mathcal{L}}_{n}$ replaced by $\underline{\mathcal{O}}_{n}$ ), it can be shown that for smooth conductors, $\boldsymbol{w}_{n}$ (and $\boldsymbol{W}_{n}$ ) are spectrally accurate approximations to the unique solutions $\boldsymbol{w}$ (and $\boldsymbol{W}$ ) in the maximum norm: $\| \boldsymbol{w}-$ $\boldsymbol{w}_{n} \|_{\infty, \partial D}=O\left(n^{-q}\right)$ (and $\left\|\boldsymbol{W}-\boldsymbol{W}_{n}\right\|_{\infty, \partial B}=O\left(n^{-q}\right)$ ) for any positive integer $q$, provided that the Lebesgue constant of $\underline{\mathcal{O}}_{n}$ is $O\left(n^{\alpha}\right)$ for some $\alpha<1$. The proof of [16, Theorem 3] is based on the known result that the Lebesgue constant of $\underline{\mathcal{L}}_{n}$ is $O(\sqrt{n})[16,22,24]$. In this work, we conjecture that the Lebesgue constant of $\underline{\mathcal{O}}_{n}$ is the same as that of $\underline{\mathcal{L}}_{n}$; a complete proof of this result similar to the theoretical analyses in $[22,24]$ is beyond the scope of this paper.

3.3. Computer implementation details. As a prerequisite for implementation, special care must be taken to avoid overflow in the evaluation of the basis functions - in particular the associated Legendre values in (3.5) when $l$ is large. We compute directly the normalized associated Legendre values

$$
\widehat{P}_{l}^{j}(x)=\sqrt{\frac{l-j}{l+j}} P_{l}^{j}(x), \quad 0 \leq l \leq n, 0 \leq j \leq l,
$$

using the relation

$$
\widehat{P}_{l}^{j}(x)=\sigma_{l}^{j} Q_{l}^{j}(x)
$$


and the recurrences

$$
\begin{aligned}
(l-j) Q_{l}^{j}(x) & =(2 l-1) x Q_{l-1}^{j}(x)-(l+j-1) Q_{l-2}^{j}(x), \quad l>j+1, \\
\sigma_{l}^{j} & =\sqrt{\frac{l-j}{l+j}} \sigma_{l-1}^{j}, \quad l>j+1,
\end{aligned}
$$

with the seeds

$$
\begin{gathered}
\sigma_{j}^{j}=1, \quad \sigma_{j+1}^{j}=\sqrt{\frac{1}{2 j+1}}, \\
Q_{j}^{j}(x)=\eta(j)\left(1-x^{2}\right)^{j / 2}, \quad Q_{j+1}^{j}(x)=x(2 l+1) Q_{j}^{j}(x), \quad \eta(j)=(2 j-1) ! ! / \sqrt{(2 j) ! .}
\end{gathered}
$$

Overflow in computing $\eta(j)$ is avoided using the stable expression

$$
\eta(j)=\prod_{\tilde{j}=1}^{j} \sqrt{\frac{2 \widetilde{j}-1}{2 \widetilde{j}}} .
$$

As in [16], we describe an efficient way to set up the $N \times N$ electromagnetic scattering matrix $\mathrm{M}$ in only $O\left(N^{2.5}\right)$ operations, where $N=3(n+1)^{2}-2$. This complexity can be reduced further by utilizing fast transforms. We also describe a parallel implementation.

Using (3.23), (3.18), (3.17), (3.14), and (3.9), we write

$$
\begin{aligned}
\mathrm{M}_{l^{\prime} j^{\prime} k^{\prime}, l j \bar{k}}= & \sum_{r=0}^{2 n+3} \sum_{s=1}^{n+2} \mu_{r}^{n+1} \nu_{s}^{n+1} \sum_{r^{\prime}=0}^{2 n^{\prime}+1} \sum_{s^{\prime}=1}^{n^{\prime}+1} \mu_{r^{\prime}}^{n^{\prime}}{ }_{s^{\prime}}^{n^{\prime}} \overline{\boldsymbol{Y}_{l^{\prime}, j^{\prime}}^{\left(k^{\prime}\right)}\left(\widehat{\boldsymbol{x}}_{r s}\right)}{ }^{T}\left[\alpha_{s^{\prime}}^{n^{\prime}} \mathcal{T}_{\widehat{\boldsymbol{x}}_{r s}} M_{1}\left(\widehat{\boldsymbol{n}}, \widehat{\boldsymbol{z}}_{r^{\prime} s^{\prime}}\right)\right. \\
& \left.+\mathcal{T}_{\widehat{\boldsymbol{x}}_{r s}} M_{2}\left(\widehat{\boldsymbol{n}}, \widehat{\boldsymbol{z}}_{r^{\prime} s^{\prime}}\right)\right] \mathcal{T}_{\widehat{\boldsymbol{x}}_{r s}} \boldsymbol{Y}_{l, j}^{(\widetilde{k})}\left(\widehat{\boldsymbol{z}}_{r^{\prime} s^{\prime}}\right)
\end{aligned}
$$

where $\widehat{\boldsymbol{x}}_{r s}=\boldsymbol{p}\left(\theta_{s}^{n+1}, \phi_{r}^{n+1}\right)$ and $\widehat{\boldsymbol{z}}_{r^{\prime} s^{\prime}}=\boldsymbol{p}\left(\theta_{s^{\prime}}^{n^{\prime}}, \phi_{r^{\prime}}^{n^{\prime}}\right)$.

Direct computation of M using (3.25) has complexity $O\left(N^{4}\right)$ because M has $O\left(N^{2}\right)$ entries, and each entry requires $O\left(N^{2}\right)$ function evaluations to approximate a double integral over $\partial B$. The efficient assembly algorithm presented below reduces this complexity to $O\left(N^{2.5}\right)$. Optimal complexity for the matrix would be $O\left(N^{2}\right)$, but the spectrally accurate nature of the algorithm compensates for the slightly higher complexity.

We write $\boldsymbol{y}_{r s}^{r^{\prime} s^{\prime}}=T_{\widehat{\boldsymbol{x}}_{r s}}^{-1} \widehat{\boldsymbol{z}}_{r^{\prime} s^{\prime}}$ so that

$$
\begin{aligned}
\mathrm{M}_{l^{\prime} j^{\prime} k^{\prime}, l j \tilde{k}}= & \sum_{r=0}^{2 n+3} \sum_{s=1}^{n+2} \mu_{r}^{n+1} \nu_{s}^{n+1} \sum_{r^{\prime}=0}^{2 n^{\prime}+1} \sum_{s^{\prime}=1}^{n^{\prime}+1} \mu_{r^{\prime}}^{n^{\prime}} \nu_{s^{\prime}}^{n^{\prime}}{\overline{\boldsymbol{Y}_{l^{\prime}, j^{\prime}}^{\left(k^{\prime}\right)}\left(\widehat{\boldsymbol{x}}_{r s}\right)}}^{T}\left[\alpha_{s^{\prime}}^{n^{\prime}} M_{1}\left(\widehat{\boldsymbol{x}}_{r s}, \widehat{\boldsymbol{y}}_{r s}^{r^{\prime} s^{\prime}}\right)\right. \\
& \left.+M_{2}\left(\widehat{\boldsymbol{x}}_{r s}, \widehat{\boldsymbol{y}}_{r s}^{r^{\prime} s^{\prime}}\right)\right] \mathcal{T}_{\widehat{\boldsymbol{x}}_{r s}} \boldsymbol{Y}_{l, j}^{(\widetilde{k})}\left(\widehat{\boldsymbol{z}}_{r^{\prime} s^{\prime}}\right)
\end{aligned}
$$

From $\left[11,(12.7 .4)\right.$, p. 341] the rotated vector spherical harmonic $\mathcal{T}_{\widehat{\boldsymbol{x}}_{r s}} \boldsymbol{Y}_{l, j}^{(\widetilde{k})}$ can be written as a linear combination of vector spherical harmonics of the same degree. As in [12], the complexity of computing $\mathrm{M}$ can be reduced by taking advantage of this property. A stable and efficient representation based on [11, (12.7.4), p. 341] using [12, 
p. 224] is

$$
\begin{aligned}
\mathcal{T}_{\widehat{\boldsymbol{x}}_{r s}} \boldsymbol{Y}_{l, j}^{(k)} & =T_{\widetilde{\boldsymbol{x}}_{r s}}^{-1} \sum_{|\widetilde{j}| \leq l} F_{s / \tilde{j} j} e^{i(j-\widetilde{j}) \phi_{r}^{n+1}} \boldsymbol{Y}_{l, \tilde{j}}^{(k)}, \\
F_{s l \widetilde{j} j} & =e^{i(j-\widetilde{j}) \pi / 2} \sum_{|\widetilde{m}| \leq l} d_{\tilde{j} \widetilde{m}}^{(l)}(\pi / 2) d_{\tilde{j} \tilde{m}}^{(l)}(\pi / 2) e^{i \widetilde{m} \theta_{s}^{n+1}},
\end{aligned}
$$

where

$$
d_{\tilde{j} j}^{(l)}(\pi / 2)=2^{\widetilde{j}}\left[\frac{(l+\widetilde{j}) !(l-\widetilde{j}) !}{(l+j) !(l-j) !}\right]^{1 / 2} P_{l+\widetilde{j}}^{(j-\widetilde{j},-j-\widetilde{j})}(0) .
$$

For given nonnegative integers $a, b$ and $s \geq 0, P_{s}^{(a, b)}(0)$ is the normalized Jacobi polynomial evaluated at zero,

$$
P_{s}^{(a, b)}(0)=2^{-s} \sum_{t=0}^{s}(-1)^{t}\left(\begin{array}{c}
s+a \\
s-t
\end{array}\right)\left(\begin{array}{c}
s+b \\
t
\end{array}\right) .
$$

When $a$ or $b$ is negative, $d_{\tilde{j} j}^{(l)}$ can be computed using the symmetry relations

$$
d_{\tilde{j} j}^{(l)}(\alpha)=(-1)^{\tilde{j}-j} d_{j \widetilde{j}}^{(l)}(\alpha)=d_{-j-\tilde{j}}^{(l)}(\alpha)=d_{\tilde{j} \tilde{j}}^{(l)}(-\alpha) .
$$

Using (3.26), (3.2), and (3.5), we get

$$
\begin{aligned}
& \mathrm{M}_{l^{\prime} j^{\prime} k^{\prime}, l j \widetilde{k}} \\
& =\sum_{r=0}^{2 n+3} \sum_{s=1}^{n+2} \mu_{r}^{n+1} \nu_{s}^{n+1} \sum_{r^{\prime}=0}^{2 n^{\prime}+1} \sum_{s^{\prime}=1}^{n^{\prime}+1} \mu_{r^{\prime}}^{n^{\prime}} \nu_{s^{\prime}}^{n^{\prime}} \sum_{t^{\prime}=1}^{3} \alpha_{l^{\prime}, j^{\prime}}^{\left(k^{\prime}, t^{\prime}\right)}\left(\theta_{s}^{n+1}\right) e^{-i j^{\prime} \phi_{r}^{n+1}} \boldsymbol{v}^{\left(t^{\prime}\right)}\left(\theta_{s}^{n+1}, \phi_{r}^{n+1}\right)^{T} \\
& \quad\left[\alpha_{s^{\prime}}^{n^{\prime}} M_{1}\left(\widehat{\boldsymbol{x}}_{r s}, \widehat{\boldsymbol{y}}_{r s}^{r^{\prime} s^{\prime}}\right)+M_{2}\left(\widehat{\boldsymbol{x}}_{r s}, \widehat{\boldsymbol{y}}_{r s}^{r^{\prime} s^{\prime}}\right)\right] \sum_{|\widetilde{j}| \leq l} F_{s / \tilde{j} j} e^{i(j-\widetilde{j}) \phi_{r}^{n+1}} \\
& \quad \sum_{t=1}^{3} \alpha_{l, \tilde{j}}^{(\widetilde{k}, t)}\left(\theta_{s^{\prime}}^{n^{\prime}}\right) e^{i \tilde{j}_{r^{\prime}}^{n^{\prime}}} T_{\widehat{\boldsymbol{x}}_{r s}}^{-1} \boldsymbol{v}^{(t)}\left(\theta_{s^{\prime}}^{n^{\prime}}, \phi_{r^{\prime}}^{n^{\prime}}\right) .
\end{aligned}
$$

The matrix M, which has $O\left(N^{2}\right)=O\left(n^{4}\right)$ entries, can be computed by successively computing the following arrays. Each array is a sum with $O(n)$ terms and depends on four labels (ignoring $\widetilde{k}$ and $\left.k^{\prime}\right)$. Thus $\mathrm{M}$ can be computed in $O\left(n^{5}\right)=O\left(N^{2.5}\right)$ operations.

$$
\begin{aligned}
\mathrm{E}_{s r s^{\prime} \tilde{j} t} & =\sum_{r^{\prime}=0}^{2 n^{\prime}+1} \mu_{r^{\prime}}^{n^{\prime}} e^{i \tilde{j} \phi_{r^{\prime}}^{n^{\prime}}}\left[\alpha_{s^{\prime}}^{n^{\prime}} M_{1}\left(\widehat{\boldsymbol{x}}_{r s}, \widehat{\boldsymbol{y}}_{r s}^{r^{\prime} s^{\prime}}\right)+M_{2}\left(\widehat{\boldsymbol{x}}_{r s}, \widehat{\boldsymbol{y}}_{r s}^{r^{\prime} s^{\prime}}\right)\right] T_{\widehat{\boldsymbol{x}}_{r s}}^{-1} \boldsymbol{v}^{(t)}\left(\theta_{s^{\prime}}^{n^{\prime}}, \phi_{r^{\prime}}^{n^{\prime}}\right), \\
\mathrm{D}_{s r l \tilde{j} \tilde{k}} & =\sum_{s^{\prime}=1}^{n^{\prime}+1} \sum_{t=1}^{3} \nu_{s^{\prime}}^{n^{\prime}} \alpha_{l, \tilde{j}}^{(\widetilde{k}, t)}\left(\theta_{s^{\prime}}^{n^{\prime}}\right) \mathrm{E}_{s r s^{\prime} \tilde{j} t}, \quad \mathrm{C}_{s r l j \tilde{k}}=\sum_{|\widetilde{j}| \leq l} F_{s l \tilde{j} j} e^{i(j-\widetilde{j}) \phi_{r}^{n+1}} \mathrm{D}_{s r l \tilde{j} \tilde{k}}, \\
\mathrm{~B}_{s j^{\prime} t^{\prime} l j \tilde{k}} & =\sum_{r=0}^{2 n+3} \mu_{r}^{n+1} e^{-i j^{\prime} \phi_{r}^{n+1}} \boldsymbol{v}^{\left(t^{\prime}\right)}\left(\theta_{s}^{n+1}, \phi_{s}^{n+1}\right)^{T} \mathrm{C}_{s r l j \tilde{k}}, \\
\mathrm{M}_{l^{\prime} j^{\prime} k^{\prime}, l j \tilde{k}} & =\sum_{s=1}^{n+2} \sum_{t^{\prime}=1}^{3} \nu_{s}^{n+1} \alpha_{l^{\prime}, j^{\prime}}^{\left(k^{\prime}, t^{\prime}\right)}\left(\theta_{s}^{n+1}\right) \mathrm{B}_{s j^{\prime} t^{\prime} l j \tilde{k}} .
\end{aligned}
$$


Once E has been used to compute D, it is discarded. Similarly, D is discarded after it has been used to compute C, and so on. The fast Fourier transform (FFT) can be used to speed up the computation of the arrays above, and for calculating $\mathrm{f}$ and $F$ in (3.23) and (3.26), respectively. As we shall demonstrate in the numerical experiments section, $5 \leq n \leq 125$ is sufficient for low to medium frequency problems because of the high-order spectral accuracy of our algorithm. Hence we can use direct or iterative solves in a single or multiprocessor environment.

For a cluster computing-type environment (with multiple processors and limited RAM for each processor), we consider a parallel implementation of the above algorithm. For a cluster computer implementation with $n_{\text {proc }}$ free CPUs, we partition the $s$ index set $\{1, \ldots, n+2\}$ into $n_{\text {proc }}$ disjoint sets $S_{p}$ for $p=1, \ldots, n_{\text {proc }}$. The array $\mathrm{B}_{s j^{\prime} t^{\prime} l j \tilde{k}}$ for $s \in S_{p}$ is computed and stored on processing node $p$.

4. High-order RCS and exterior field approximations. In this section, we follow a variant of the approach in [16] to describe a fully discrete high-order approximation to the monostatic and bistatic RCS of the perfect conductor $D$, and the exterior electromagnetic fields. We use the spectrally accurate numerical solution $\boldsymbol{W}_{n}$ of (3.21)-(3.23) for this purpose.

4.1. Spectrally accurate RCS computations. The monostatic and bistatic RCS measurements in (1.2) and (1.7) require high-order approximations of the electric far field. Using (2.6), the electric far field corresponding to the unique solution $\boldsymbol{w}=$ $\mathcal{P} \boldsymbol{w}$ of (2.3) can be written as

$$
\begin{aligned}
& \boldsymbol{E}_{\infty}(\widehat{\boldsymbol{x}})=\frac{i k}{4 \pi} \int_{\partial D} e^{-i k \widehat{\boldsymbol{x}} \cdot \boldsymbol{y}} \widehat{\boldsymbol{x}} \times \mathcal{P}(\boldsymbol{y}) \boldsymbol{w}(\boldsymbol{y}) d s(\boldsymbol{y}) \\
& =\int_{\partial B} \widehat{M}_{\widehat{\boldsymbol{x}}}(\widehat{\boldsymbol{y}}) \boldsymbol{W}(\widehat{\boldsymbol{y}}) d s(\widehat{\boldsymbol{y}}), \quad \widehat{\boldsymbol{x}} \in \partial B,
\end{aligned}
$$

where for each fixed $\widehat{\boldsymbol{x}} \in \partial B$, the smooth function $\widehat{M}_{\widehat{\boldsymbol{x}}}$ is defined by

$$
\widehat{M}_{\widehat{\boldsymbol{x}}}(\widehat{\boldsymbol{y}}) \boldsymbol{W}(\widehat{\boldsymbol{y}})=\frac{i k}{4 \pi} J(\widehat{\boldsymbol{y}}) e^{-i k \widehat{\boldsymbol{x}} \cdot \boldsymbol{q}(\widehat{\boldsymbol{y}})} \widehat{\boldsymbol{x}} \times \mathcal{P}(\boldsymbol{q}(\widehat{\boldsymbol{y}})) \boldsymbol{W}(\widehat{\boldsymbol{y}}), \quad \widehat{\boldsymbol{y}} \in \partial B,
$$

and $\boldsymbol{W}$ is the solution of (2.23).

Using the approximation $\boldsymbol{W}_{n}$ to $\boldsymbol{W}$ given by (3.21)-(3.23), our fully discrete approximation $\boldsymbol{E}_{n, \infty}(\widehat{\boldsymbol{x}})$ to $\boldsymbol{E}_{\infty}(\widehat{\boldsymbol{x}})$ for $\widehat{\boldsymbol{x}} \in \partial B$ is given by

$$
\begin{aligned}
\boldsymbol{E}_{n, \infty}(\widehat{\boldsymbol{x}}) & =\int_{\partial B} \underline{\mathcal{O}}_{n}\left\{\widehat{M}_{\widehat{\boldsymbol{x}}}(\cdot) \boldsymbol{W}_{n}(\cdot)\right\}(\widehat{\boldsymbol{y}}) d s(\widehat{\boldsymbol{y}}) \\
& =\sum_{l=0}^{n} \sum_{|j| \leq l} \sum_{\widetilde{k}=1}^{3} \mathrm{w}_{l j \widetilde{k}} \int_{\partial B} \underline{\mathcal{O}}_{n}\left\{\widehat{M}_{\widehat{\boldsymbol{x}}}(\cdot) \boldsymbol{Y}_{l, j}^{(\widetilde{k})}(\cdot)\right\}(\widehat{\boldsymbol{y}}) d s(\widehat{\boldsymbol{y}}),
\end{aligned}
$$

where, for convenience, we set $\mathrm{w}_{001}=\mathrm{w}_{002}=0$. Using (3.10) and (3.7),

$$
\boldsymbol{E}_{n, \infty}(\widehat{\boldsymbol{x}})=\sum_{l=0}^{n} \sum_{|j| \leq l} \sum_{\widetilde{k}=1}^{3} \mathrm{w}_{l j} \sum_{r=0}^{2 n+3} \sum_{s=1}^{n+2} \mu_{r}^{n+1} \nu_{s}^{n+1} A_{r s} \widehat{M}_{\widehat{\boldsymbol{x}}}\left(\widehat{\boldsymbol{z}}_{r s}^{n+1}\right) \boldsymbol{Y}_{l, j}^{(\widetilde{k})}\left(\widehat{\boldsymbol{z}}_{r s}^{n+1}\right),
$$

where

$$
A_{r s}=\sum_{l^{\prime}=0}^{n} \sum_{\left|j^{\prime}\right| \leq l} \sum_{k^{\prime}=1}^{3}\left(\int_{\partial B} \boldsymbol{Y}_{l^{\prime}, j^{\prime}}^{\left(k^{\prime}\right)}(\widehat{\boldsymbol{y}}) d s(\widehat{\boldsymbol{y}})\right){\overline{\boldsymbol{Y}_{l^{\prime}, j^{\prime}}^{\left(k^{\prime}\right)}\left(\widehat{\boldsymbol{z}}_{r s}^{n+1}\right)}}^{T}
$$


Only the integrals $\left(l^{\prime}, j^{\prime}, k^{\prime}\right) \in\{(0,0,3),(1, \pm 1,1),(1, \pm 1,2)\}$ are nonzero, and they can be easily computed analytically. Proceeding as in [16], we have

$$
\begin{aligned}
\boldsymbol{E}_{n, \infty}(\widehat{\boldsymbol{x}}) & =\sum_{l=0}^{n} \sum_{|j| \leq l} \sum_{\widetilde{k}=1}^{3} \mathrm{w}_{l j \widetilde{k}} \widehat{\boldsymbol{M}}_{l j \widetilde{k}}^{n+1}(\widehat{\boldsymbol{x}}), \\
\widehat{\boldsymbol{M}}_{l j \widetilde{k}}^{n+1}(\widehat{\boldsymbol{x}}) & =\sum_{r=0}^{2 n+3} \sum_{s=1}^{n+2} \mu_{r}^{n+1} \nu_{s}^{n+1} A_{r s} \widehat{M}_{\widehat{\boldsymbol{x}}}\left(\widehat{\boldsymbol{z}}_{r s}^{n+1}\right) \boldsymbol{Y}_{l, j}^{(\widetilde{k})}\left(\widehat{\boldsymbol{z}}_{r s}^{n+1}\right) .
\end{aligned}
$$

The quantities $\widehat{\boldsymbol{M}}_{l j \tilde{k}}^{n+1}$ do not depend on $\boldsymbol{W}_{n}$ and can be precomputed independently. As in [16, Theorem 3], assuming the conjecture of section 3.2, we can prove that $\boldsymbol{E}_{n, \infty}$ is a spectrally accurate approximation to $\boldsymbol{E}_{\infty}$.

The approximation $\sigma_{n}^{\mathrm{dB}}$ to the RCS $\sigma^{\mathrm{dB}}$ is obtained by replacing $\boldsymbol{E}_{\infty}$ by $\boldsymbol{E}_{n, \infty}$ in (1.2) and (1.7). That is, we compute the spectrally accurate approximation to the RCS of the perfect conductor $D \subseteq \mathbb{R}^{3}$ using the fully discrete formulas

$$
\begin{aligned}
\sigma_{n}^{\mathrm{dB}}\left(\widehat{\boldsymbol{x}} ; \widehat{\boldsymbol{d}}_{0}\right) & =10 \log _{10}\left(4 \pi\left|\boldsymbol{E}_{n, \infty}(\widehat{\boldsymbol{x}})\right|^{2} / k^{2}\right), \\
\sigma_{n}^{\mathrm{dB}}\left(\widehat{\boldsymbol{x}}, \widehat{\boldsymbol{p}}_{1} ; \widehat{\boldsymbol{d}}_{0}\right) & =10 \log _{10}\left(4 \pi\left|\boldsymbol{E}_{n, \infty}(\widehat{\boldsymbol{x}}) \cdot \widehat{\boldsymbol{p}}_{1}\right|^{2} / k^{2}\right), \quad \widehat{\boldsymbol{x}} \in \partial B .
\end{aligned}
$$

For each $\widehat{\boldsymbol{x}} \in \partial B$, direct computation of $\boldsymbol{E}_{n, \infty}(\widehat{\boldsymbol{x}})$ has complexity $O\left(n^{4}\right)$ because $\widehat{\boldsymbol{M}}^{n+1}$ has $O\left(n^{2}\right)$ entries, and each entry requires $O\left(n^{2}\right)$ function evaluations to approximate an integral over $\partial B$. However, $\boldsymbol{E}_{n, \infty}(\widehat{\boldsymbol{x}})$ can be computed in $O\left(n^{3}\right)$ operations by successively computing the quantities below to obtain $\widehat{\boldsymbol{M}}^{n+1}$ :

$$
\begin{aligned}
\widehat{\boldsymbol{B}}_{s j t}^{n+1}(\widehat{\boldsymbol{x}}) & =\sum_{r=0}^{2 n+3} \mu_{r}^{n+1} A_{r s} \widehat{M}_{\widehat{\boldsymbol{x}}}\left(\widehat{\boldsymbol{z}}_{r s}^{n+1}\right) e^{i j \phi_{r}^{n+1}} \boldsymbol{v}^{t}\left(\theta_{s}^{n+1}, \phi_{r}^{n+1}\right), \\
\widehat{\boldsymbol{M}}_{l j \widetilde{k}}^{n+1}(\widehat{\boldsymbol{x}}) & =\sum_{s=1}^{n+2} \sum_{t=1}^{3} \nu_{s}^{n+1} \alpha_{l, j}^{(\widetilde{k}, t)}\left(\theta_{s}^{n+1}\right) \widehat{\boldsymbol{B}}_{s j t}^{n+1}(\widehat{\boldsymbol{x}}) .
\end{aligned}
$$

The computation of $\widehat{\boldsymbol{B}}^{n+1}$ can be accelerated using the FFT. Once $\widehat{\boldsymbol{M}}^{n+1}(\widehat{\boldsymbol{x}})$ has been evaluated, it can be used for computation of the far field induced by many incident directions in the monostatic RCS approximations.

4.2. Spectrally accurate exterior field computations. We compute a fully discrete approximation to the electric field $\boldsymbol{E}(\boldsymbol{x})$ in the exterior region, given by (2.4), using the approach similar to the far field computation in section 4.1, but we replace $\widehat{M}_{\widehat{\boldsymbol{x}}}$ by $\widetilde{M}_{\boldsymbol{x}}$, where

$$
\widetilde{M}_{\boldsymbol{x}}(\widehat{\boldsymbol{y}}) \boldsymbol{W}(\widehat{\boldsymbol{y}})=J(\widehat{\boldsymbol{y}}) \operatorname{grad}_{\boldsymbol{x}} \Phi(\boldsymbol{x}, \boldsymbol{q}(\widehat{\boldsymbol{y}})) \times \mathcal{P}(\boldsymbol{q}(\widehat{\boldsymbol{y}})) \boldsymbol{W}(\widehat{\boldsymbol{y}}), \quad \widehat{\boldsymbol{y}} \in \partial B .
$$

We have used (2.4) and the identity curl $\{\phi \boldsymbol{v}\}=\phi \operatorname{curl} \boldsymbol{v}+\operatorname{grad} \phi \times \boldsymbol{v}$, to derive $\widetilde{M}_{\boldsymbol{x}}$. The fully discrete approximation $\boldsymbol{E}_{n}(\boldsymbol{x})$ to $\boldsymbol{E}(\boldsymbol{x})$ for $\boldsymbol{x} \in \mathbb{R}^{3} \backslash \bar{D}$ is then

$$
\begin{aligned}
\boldsymbol{E}_{n}(\boldsymbol{x}) & =\sum_{l=0}^{n} \sum_{|j| \leq l} \sum_{\widetilde{k}=1}^{3} \mathrm{w}_{l j \widetilde{k}} \widetilde{\boldsymbol{M}}_{l j \widetilde{k}}^{n+1}(\boldsymbol{x}), \\
\widetilde{\boldsymbol{M}}_{l j \widetilde{k}}^{n+1}(\boldsymbol{x}) & =\sum_{r=0}^{2 n+3} \sum_{s=1}^{n+2} \mu_{r}^{n+1} \nu_{s}^{n+1} A_{r s} \widetilde{M}_{\boldsymbol{x}}\left(\widehat{\boldsymbol{z}}_{r s}^{n+1}\right) \boldsymbol{Y}_{l, j}^{(\widetilde{k})}\left(\widehat{\boldsymbol{z}}_{r s}^{n+1}\right) .
\end{aligned}
$$

These computations can be accelerated as in section 4.1. 
5. Simulated RCS and exterior electromagnetic fields. We consider the RCS simulation problem (1.1)-(1.7) for various test obstacles including the benchmark radar target cone-sphere [30] and a biologically interesting erythrocyte in standard oblate and in oval of Cassini prolate form [19,31]. For an incident wave with incident polar and azimuthal angles $\theta$ and $\phi$, the direction of the plane wave is given by $\widehat{\boldsymbol{d}}_{0}=-(\sin \theta \cos \phi, \sin \theta \sin \phi, \cos \theta)^{T}$. The incident wave is then vertically polarized when $\widehat{\boldsymbol{p}}_{0}=(\cos \theta \cos \phi, \cos \theta \sin \phi,-\sin \theta)^{T}$ and horizontally polarized when $\widehat{\boldsymbol{p}}_{0}=(-\sin \phi, \cos \phi, 0)^{T}[27$, pp. 8-9]. Throughout this section we denote the RCS $\sigma_{n}^{\mathrm{dB}}\left(\boldsymbol{p}(\theta, \phi) ; \widehat{\boldsymbol{d}}_{0}\right)$ in $(4.5)$ by $\sigma_{n}^{\mathrm{dB}}(\theta, \phi)$.

Given the spatial component of an electric field, denoted by $\boldsymbol{E}$, in $\mathbb{R}^{3} \backslash \bar{D}$, the corresponding time harmonic electric field is given by

$$
\mathcal{E}(\boldsymbol{x}, t)=\frac{1}{\sqrt{\epsilon_{0}}} \operatorname{Re}\left\{\boldsymbol{E}(\boldsymbol{x}) e^{-2 \pi i \omega t}\right\}, \quad \boldsymbol{x} \in \mathbb{R}^{3} \backslash \bar{D} .
$$

In our numerical experiments we simulate the monostatic and bistatic RCS and exterior electromagnetic fields induced by smooth convex and nonconvex obstacles, and by the cone-sphere, a benchmark radar target that has conical singularities. The smooth obstacles are a sphere, $\mathbf{s p h}($ siz_obs); a beehive shape, hive(siz_obs); a Cassini shape in prolate form, $\mathbf{c a s}\left(s i z_{-} o b s\right)$; an erythrocyte, eryth(siz_obs); and a submarine shape, $\mathbf{s u b}\left(\right.$ siz_obs$_{-}$). The nonsmooth benchmark radar target is the perfectly conducting cone-sphere, cone_sphere(siz_obs). Here siz_obs is the diameter of the obstacle. These obstacles are shown in Figures 1-3. The erythrocyte is constructed according to [31, equation (3)]. As described in [19,31], the biconcave cross section of the erythrocyte has the shape of the Cassini obstacle in two-dimensional form. The cone-sphere is constructed according to [30]. The hull of the submarine is constructed, as in [18], from an ellipsoid with aspect ratio 10:1:1. The cone-sphere and submarine have higher aspect ratios than the other obstacles considered.

For a fixed positive integer $n$, the linear system we need to solve to achieve the reported accuracy has $3(n+1)^{2}-2$ unknowns in general. For spherical scattering the linear system has $2(n+1)^{2}-2$ unknowns. The maximum $n$ we used in all the experiments is only 125 . Thus we are able to present results obtained with both direct and iterative linear system solvers. In the case of iterative solvers, we use GMRES preconditioned with the inverse of the diagonal part of our matrix. For our experiments, we used a cluster with $2 \mathrm{GHz}$ Dual-core Opteron (DcOp) processors. DcOp CPU time mentioned in this paper is for the combined set up, GMRES solve, and error calculation phases of the algorithm.

In the case of plane wave scattering by a sphere, an analytical representation of the scattered field is given by the Mie series. For the sphere case our choice of frequency for the incident wave (and hence of the electromagnetic scattering problem) is motivated by those considered in established electromagnetic scattering algorithms Fastscat [5] and FISC [25]. To compare the efficiency of our algorithm with [5, 25], in the tabulated results we use the RMS error (denoted by $\epsilon_{\mathrm{dB}}$ ) in the bistatic RCS, measured in decibels per square meter. The RMS error is defined as

$$
\epsilon_{\mathrm{dB}}=\left\{\frac{1}{4 \pi} \int_{\partial B}\left[\sigma^{\mathrm{dB}}(\widehat{\boldsymbol{x}})-\sigma_{n}^{\mathrm{dB}}(\widehat{\boldsymbol{x}})\right]^{2} d s(\widehat{\boldsymbol{x}})\right\}^{1 / 2},
$$

where $\sigma^{\mathrm{dB}}$ and $\sigma_{n}^{\mathrm{dB}}$ are, respectively, defined by (1.2)-(1.7) and (4.5). We numerically approximate the integral in $\epsilon_{\mathrm{dB}}$ using over 1300 quadrature points on $\partial B$. 


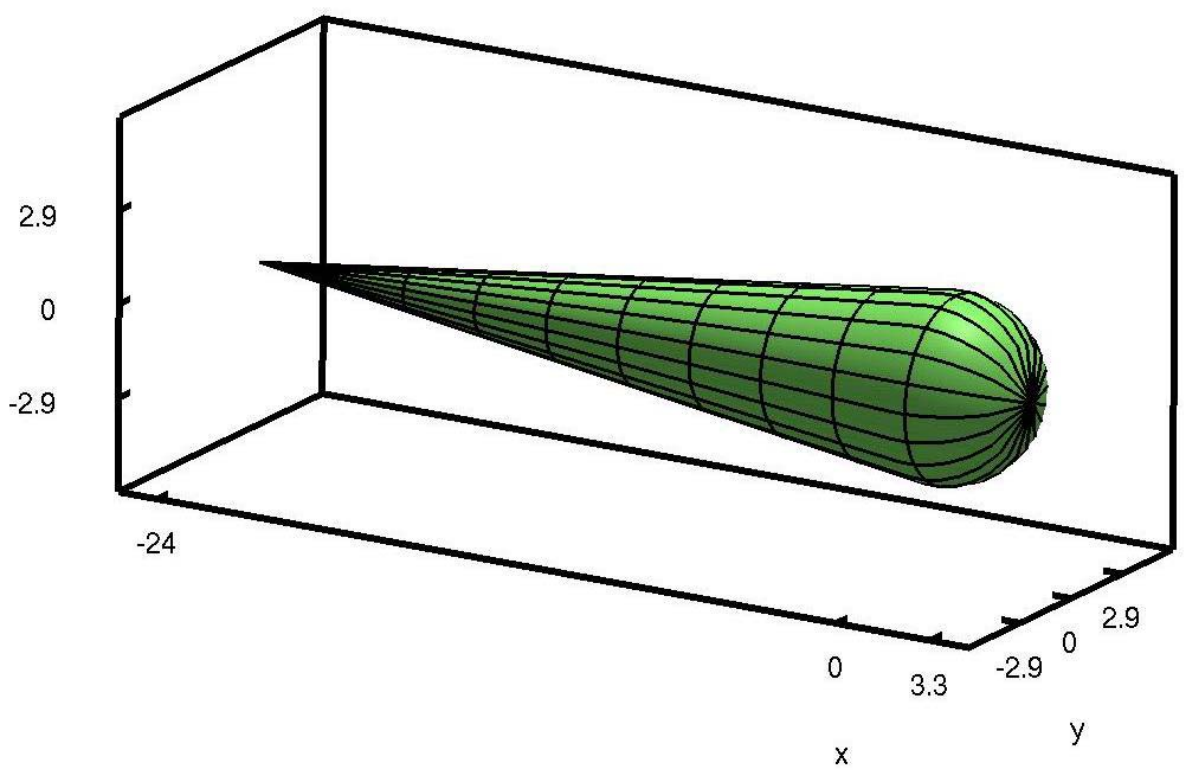

FIG. 1. Cone-sphere.
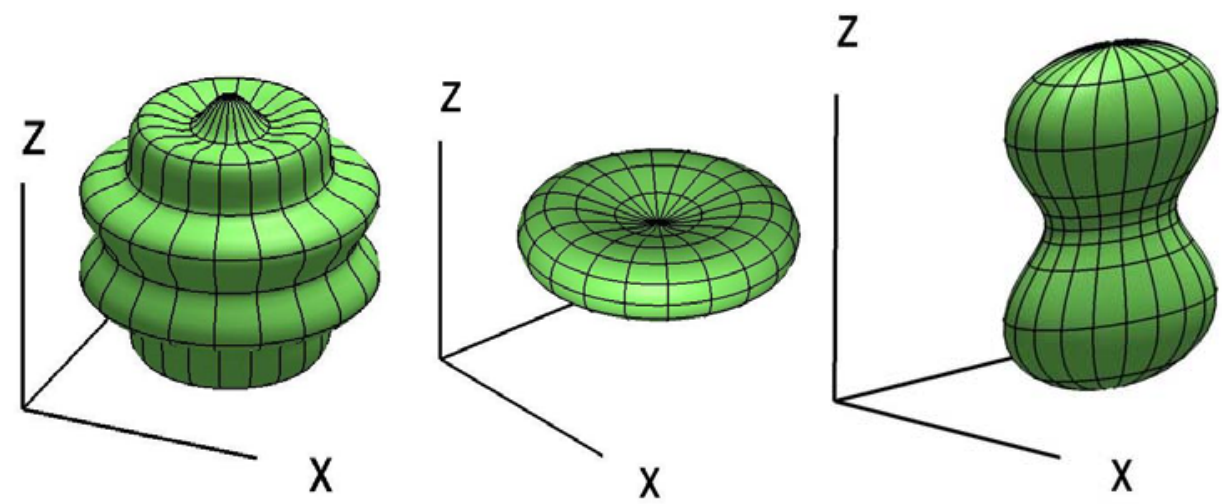

FIG. 2. Hive, Erythrocyte, and Cassini.

To demonstrate the spectral convergence of our method for plane wave scattering by nonspherical perfect conductors, we tabulate the monostatic or bistatic RCS at various receiver direction angles. Similar convergence studies for acoustic scattering in two and three dimensions are, respectively, in [8, p. 72] and [12, p. 234].

For the erythrocyte an incident wave with wavelength $632.8 \mathrm{~nm}$ (or one tenth the diameter of the erythrocyte) is of considerable recent interest [19, 31]. Hence, for RCS computations of a fixed target, we choose two incident waves: one with wavelength equal to the diameter of the target, and the other with wavelength one tenth the diameter of the target. We observed similar performance for various other targets considered in [16].

The results in Tables 1-15 demonstrate the spectral accuracy of our algorithm. 


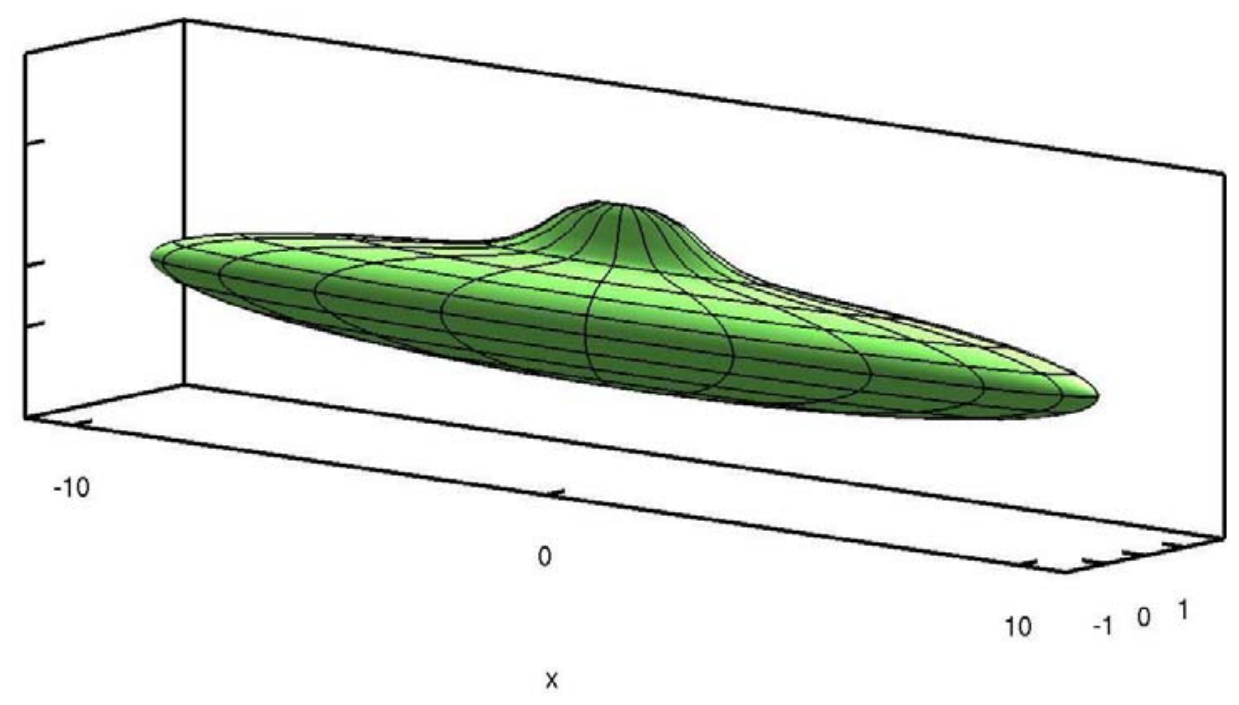

y

FiG. 3. Submarine.

As would be expected, the spherical perfect conductor requires fewer unknowns than the high aspect-ratio and nonconvex obstacles. In general, the number of unknowns depends on the shape and electromagnetic size siz_obs/ $\lambda$ of the conductors, where $\lambda=2 \pi / k=c / \omega$ is the wavelength. The cone-sphere possesses a conical singularity. This nonsmoothness in the object surface precludes spectral accuracy, but our efficient algorithm is able to produce visually accurate results using only a few tens of thousands of unknowns.

We conclude this work by presenting visualizations of RCS values and exterior fields of all the described obstacles simulated using the medium frequency electromagnetic scattering model described in the introduction (see Figures 4-9). The left colorbar values in the figures correspond to the intensity of the surface current. The RCS plots (for all geometries considered in this paper) are given in polar form. For comparison of the experimental and simulated RCS for the cone-sphere benchmark target in [30], we also give the cone-sphere RCS plot in Cartesian form. (All figures in this paper are also at http://www.mines.edu/ mganesh/GH_pap2_figures/ GH_RCS_figures.html, to facilitate the viewing of larger pictures.)

TABLE 1

Convergence of bistatic $R C S$ for $\mathbf{s p h}(\lambda)$ at $299.8 \mathrm{MHz}$ for various observed angles ( $H$ - $H$ polarization).

\begin{tabular}{ccccc}
\hline$n$ & $\sigma_{n}^{\mathrm{dB}}\left(\pi / 2,0.0^{\circ}\right)$ & $\sigma_{n}^{\mathrm{dB}}\left(\pi / 2,59.9^{\circ}\right)$ & $\sigma_{n}^{\mathrm{dB}}\left(\pi / 2,120.1^{\circ}\right)$ & $\sigma_{n}^{\mathrm{dB}}\left(\pi / 2,180.0^{\circ}\right)$ \\
\hline 5 & -0.236515555 & -2.364344683 & -2.364344683 & -0.236515555 \\
15 & -2.261565541 & 1.633695466 & 4.172258451 & 9.660449813 \\
25 & -2.261565541 & 1.633695466 & 4.172258451 & 9.660449813 \\
\hline
\end{tabular}


TABLE 2

Convergence of bistatic $R C S$ for $\mathbf{s p h}(10 \lambda)$ at $3.0 \mathrm{GHz}$ for various observed angles ( $H$ - $H$ polarization).

\begin{tabular}{ccccc}
\hline$n$ & $\sigma_{n}^{\mathrm{dB}}\left(\pi / 2,0.0^{\circ}\right)$ & $\sigma_{n}^{\mathrm{dB}}\left(\pi / 2,59.9^{\circ}\right)$ & $\sigma_{n}^{\mathrm{dB}}\left(\pi / 2,120.1^{\circ}\right)$ & $\sigma_{n}^{\mathrm{dB}}\left(\pi / 2,180.0^{\circ}\right)$ \\
\hline 25 & 14.46493344 & 6.810093006 & 6.773474395 & 23.32394310 \\
35 & -1.071614837 & -1.100478363 & -1.356902718 & 28.98841095 \\
45 & -0.963851816 & -1.146497199 & -1.226580441 & 28.98854327 \\
55 & -0.963851776 & -1.146497197 & -1.226580462 & 28.98854327 \\
\hline
\end{tabular}

TABLE 3

Convergence of bistatic RCS for hive $(\lambda)$ at $136.3 \mathrm{MHz}$ for various observed angles ( $H$ - $H$ polarization).

\begin{tabular}{ccccc}
\hline$n$ & $\sigma_{n}^{\mathrm{dB}}\left(\pi / 2,0.0^{\circ}\right)$ & $\sigma_{n}^{\mathrm{dB}}\left(\pi / 2,59.9^{\circ}\right)$ & $\sigma_{n}^{\mathrm{dB}}\left(\pi / 2,120.1^{\circ}\right)$ & $\sigma_{n}^{\mathrm{dB}}\left(\pi / 2,180.0^{\circ}\right)$ \\
\hline 45 & 2.288774212 & 7.726946931 & 11.02191340 & 15.47284506 \\
55 & 2.289516548 & 7.727529734 & 11.02241885 & 15.47305847 \\
65 & 2.289501331 & 7.727506252 & 11.02243226 & 15.47305073 \\
75 & 2.289525690 & 7.727521530 & 11.02244076 & 15.47305490 \\
\hline
\end{tabular}

TABLE 4

Convergence of bistatic $R C S$ for hive $(10 \lambda)$ at $1.4 \mathrm{GHz}$ for various observed angles ( $H$ - $H$ polarization).

\begin{tabular}{ccccc}
\hline$n$ & $\sigma_{n}^{\mathrm{dB}}\left(\pi / 2,0.0^{\circ}\right)$ & $\sigma_{n}^{\mathrm{dB}}\left(\pi / 2,59.9^{\circ}\right)$ & $\sigma_{n}^{\mathrm{dB}}\left(\pi / 2,120.1^{\circ}\right)$ & $\sigma_{n}^{\mathrm{dB}}\left(\pi / 2,180.0^{\circ}\right)$ \\
\hline 75 & 12.21545719 & 0.995924035 & 1.630599480 & 34.71680622 \\
85 & 12.21598424 & 0.994537745 & 1.629710655 & 34.71678624 \\
95 & 12.21595582 & 0.994581511 & 1.629615318 & 34.71679053 \\
105 & 12.21595283 & 0.994589476 & 1.629597682 & 34.71679052 \\
\hline
\end{tabular}

TABLE 5

Convergence of bistatic $R C S$ for $\operatorname{cas}(\lambda)$ at $299.8 \mathrm{MHz}$ for various observed angles ( $H$ - $H$ polarization).

\begin{tabular}{ccccc}
\hline$n$ & $\sigma_{n}^{\mathrm{dB}}\left(\pi / 2,0.0^{\circ}\right)$ & $\sigma_{n}^{\mathrm{dB}}\left(\pi / 2,59.9^{\circ}\right)$ & $\sigma_{n}^{\mathrm{dB}}\left(\pi / 2,120.1^{\circ}\right)$ & $\sigma_{n}^{\mathrm{dB}}\left(\pi / 2,180.0^{\circ}\right)$ \\
\hline 15 & 5.943260950 & 2.430092594 & 2.390502200 & 8.643517631 \\
25 & 5.943542313 & 2.430600826 & 2.391709693 & 8.644488008 \\
35 & 5.943543764 & 2.430596856 & 2.391702443 & 8.644485832 \\
45 & 5.943543753 & 2.430596874 & 2.391702493 & 8.644485855 \\
\hline
\end{tabular}

TABle 6

Convergence of bistatic RCS for $\operatorname{cas}(10 \lambda)$ at $3.0 \mathrm{GHz}$ for various observed angles ( $H$ - $H$ polarization).

\begin{tabular}{lcccc}
\hline$n$ & $\sigma_{n}^{\mathrm{dB}}\left(\pi / 2,0.0^{\circ}\right)$ & $\sigma_{n}^{\mathrm{dB}}\left(\pi / 2,59.9^{\circ}\right)$ & $\sigma_{n}^{\mathrm{dB}}\left(\pi / 2,120.1^{\circ}\right)$ & $\sigma_{n}^{\mathrm{dB}}\left(\pi / 2,180.0^{\circ}\right)$ \\
\hline 75 & 5.254445300 & -2.943734543 & -1.852919510 & 27.01202739 \\
85 & 5.254455451 & -2.946501042 & -1.852890342 & 27.01202909 \\
95 & 5.254455694 & -2.946503971 & -1.852892016 & 27.01202911 \\
105 & 5.254455695 & -2.946503957 & -1.852891993 & 27.01202911 \\
\hline
\end{tabular}

TABle 7

Convergence of bistatic RCS for $\operatorname{eryth}(\lambda)$ at $47.6 \mathrm{THz}$ for various observed angles ( $V$ - $V$ polarization).

\begin{tabular}{ccccc}
\hline$n$ & $\sigma_{n}^{\mathrm{dB}}\left(0.0^{\circ}, 0\right)$ & $\sigma_{n}^{\mathrm{dB}}\left(59.9^{\circ}, 0\right)$ & $\sigma_{n}^{\mathrm{dB}}\left(120.1^{\circ}, 0\right)$ & $\sigma_{n}^{\mathrm{dB}}\left(180.0^{\circ}, 0\right)$ \\
\hline 35 & -95.26308767 & -104.7283471 & -104.7901000 & -93.60662218 \\
45 & -95.26308773 & -104.7283471 & -104.7900999 & -93.60662217 \\
55 & -95.26308774 & -104.7283471 & -104.7900999 & -93.60662217 \\
\hline
\end{tabular}


TABLE 8


polarization).

\begin{tabular}{ccccc}
\hline$n$ & $\sigma_{n}^{\mathrm{dB}}\left(0.0^{\circ}, 0\right)$ & $\sigma_{n}^{\mathrm{dB}}\left(59.9^{\circ}, 0\right)$ & $\sigma_{n}^{\mathrm{dB}}\left(120.1^{\circ}, 0\right)$ & $\sigma_{n}^{\mathrm{dB}}\left(180.0^{\circ}, 0\right)$ \\
\hline 55 & -84.15631909 & -107.9716243 & -108.5694295 & -75.08585121 \\
65 & -84.15631929 & -107.9716914 & -108.5694124 & -75.08585126 \\
75 & -84.15631929 & -107.9716925 & -108.5694122 & -75.08585127 \\
85 & -84.15631929 & -107.9716925 & -108.5694121 & -75.08585127 \\
\hline
\end{tabular}

TABLE 9

Convergence of bistatic RCS for $\mathbf{s u b}(\lambda)$ at $15.0 \mathrm{MHz}$ for various observed angles ( $H$ - $H$ polarization).

\begin{tabular}{ccccc}
\hline$n$ & $\sigma_{n}^{\mathrm{dB}}\left(\pi / 2,0.0^{\circ}\right)$ & $\sigma_{n}^{\mathrm{dB}}\left(\pi / 2,59.9^{\circ}\right)$ & $\sigma_{n}^{\mathrm{dB}}\left(\pi / 2,120.1^{\circ}\right)$ & $\sigma_{n}^{\mathrm{dB}}\left(\pi / 2,180.0^{\circ}\right)$ \\
\hline 65 & -9.373283806 & -21.40500784 & -1.314272969 & -21.59519924 \\
75 & -9.373250028 & -21.40486747 & -1.314287529 & -21.59504994 \\
85 & -9.373233746 & -21.40481200 & -1.314291518 & -21.59501892 \\
95 & -9.373225148 & -21.40478591 & -1.314292190 & -21.59502340 \\
\hline
\end{tabular}

TABLE 10

Convergence of bistatic RCS for $\mathbf{s u b}(10 \lambda)$ at $149.9 \mathrm{MHz}$ for various observed angles ( $H$ - $H$ polarization).

\begin{tabular}{ccccc}
\hline$n$ & $\sigma_{n}^{\mathrm{dB}}\left(\pi / 2,0.0^{\circ}\right)$ & $\sigma_{n}^{\mathrm{dB}}\left(\pi / 2,59.9^{\circ}\right)$ & $\sigma_{n}^{\mathrm{dB}}\left(\pi / 2,120.1^{\circ}\right)$ & $\sigma_{n}^{\mathrm{dB}}\left(\pi / 2,180.0^{\circ}\right)$ \\
\hline 95 & -7.383375719 & -5.795755529 & 3.729626885 & 11.10711087 \\
105 & -7.383132611 & -5.795728691 & 3.729627319 & 11.10710545 \\
115 & -7.383109088 & -5.795729699 & 3.729618894 & 11.10710286 \\
125 & -7.383096050 & -5.795728967 & 3.729613469 & 11.10710325 \\
\hline
\end{tabular}

TABLE 11

Convergence of monostatic RCS for $\mathbf{c o n e} \_\mathbf{s p h}(\lambda)$ at $435.10 \mathrm{MHz}$ for various observed angles ( $V$ - $V$ polarization).

\begin{tabular}{ccccc}
\hline$n$ & $\sigma_{n}^{\mathrm{dB}}\left(\pi / 2,0.0^{\circ}\right)$ & $\sigma_{n}^{\mathrm{dB}}\left(\pi / 2,59.9^{\circ}\right)$ & $\sigma_{n}^{\mathrm{dB}}\left(\pi / 2,120.1^{\circ}\right)$ & $\sigma_{n}^{\mathrm{dB}}\left(\pi / 2,180.0^{\circ}\right)$ \\
\hline 65 & -19.88430237 & -15.40047977 & -15.75751788 & -19.98539600 \\
75 & -19.80017082 & -15.37370602 & -15.72647896 & -19.87851607 \\
85 & -19.73890236 & -15.35379602 & -15.70245764 & -19.79750201 \\
95 & -19.68627108 & -15.33603751 & -15.68130174 & -19.72927847 \\
\hline
\end{tabular}

TABLE 12


( $V$ - $V$ polarization).

\begin{tabular}{ccccc}
\hline$n$ & $\sigma_{n}^{\mathrm{dB}}\left(\pi / 2,0.0^{\circ}\right)$ & $\sigma_{n}^{\mathrm{dB}}\left(\pi / 2,59.9^{\circ}\right)$ & $\sigma_{n}^{\mathrm{dB}}\left(\pi / 2,120.1^{\circ}\right)$ & $\sigma_{n}^{\mathrm{dB}}\left(\pi / 2,180.0^{\circ}\right)$ \\
\hline 95 & -19.24152606 & -15.95918276 & -30.14099402 & -29.16753533 \\
105 & -19.44876757 & -15.98494703 & -30.17249637 & -29.38451260 \\
115 & -19.47098794 & -16.03618277 & -30.28330884 & -28.94057167 \\
125 & -19.43245611 & -16.08937090 & -30.41726902 & -28.72183348 \\
\hline
\end{tabular}


TABLE 13

Performance of FastScat, FISC [25, p. 28] and GH (present). Scattering of a plane wave by a sphere of diameter $5.4 \lambda$.

\begin{tabular}{ccccc}
\hline Algorithm & Unknowns & Computer/CPU & CPU time & $\epsilon_{\mathrm{dB}}$ \\
\hline $\begin{array}{c}\text { FastScat } \\
\text { (Nystrom) }\end{array}$ & 5400 & Sparc 10 & 33688 secs & $9.7 \mathrm{e}-02$ \\
$\begin{array}{c}\text { FastScat } \\
\text { (Galerkin) }\end{array}$ & 5400 & Sparc 10 & 74956 secs & $2.1 \mathrm{e}-02$ \\
$\begin{array}{c}\text { FISC } \\
\text { (Galerkin) }\end{array}$ & 5880 & Sparc 10 & 37205 secs & $7.2 \mathrm{e}-01$ \\
GH & $1350(n=25)$ & $1 \times$ DcOp & 39 secs & $1.1 \mathrm{e}-06$ \\
\hline
\end{tabular}

TABLE 14

Performance of FISC [25, p. 28] and GH (present). Scattering of a plane wave by a sphere of diameter $24 \lambda$.

\begin{tabular}{ccccc}
\hline Algorithm & Unknowns & Computer/CPU & CPU time & $\epsilon_{\mathrm{dB}}$ \\
\hline FISC & 602112 & $\begin{array}{c}\text { SGI Power } \\
\text { Challenge R8000 }\end{array}$ & 12 hours & $3.0 \mathrm{e}-01$ \\
& & $2 \times$ DcOp & 0.80 hours & $5.7 \mathrm{e}-02$ \\
GH & $13120(n=80)$ & $4 \times$ DcOp & 0.48 hours & $5.7 \mathrm{e}-02$ \\
GH & $13120(n=80)$ & $4 \times$ DcOp & 0.80 hours & $1.5 \mathrm{e}-05$ \\
GH & $15840(n=88)$ & 4 &
\end{tabular}

TABLE 15

CPU time for computing monostatic and bistatic RCS with direct solves for 1202 sampling directions.

\begin{tabular}{lcccc}
\hline Geometry & Unknowns & Type & CPU & CPU time \\
\hline eryth $(\lambda)$ & $6348(n=45)$ & Bistatic & $2 \times$ DcOp & 8.30 mins \\
eryth $(\lambda)$ & $6348(n=45)$ & Monostatic & $2 \times$ DcOp & 12.22 mins \\
eryth $(10 \lambda)$ & $22188(n=85)$ & Bistatic & $8 \times$ DcOp & 1.13 hours \\
eryth $(10 \lambda)$ & $22188(n=85)$ & Monostatic & $8 \times$ DcOp & 2.11 hours \\
\hline
\end{tabular}



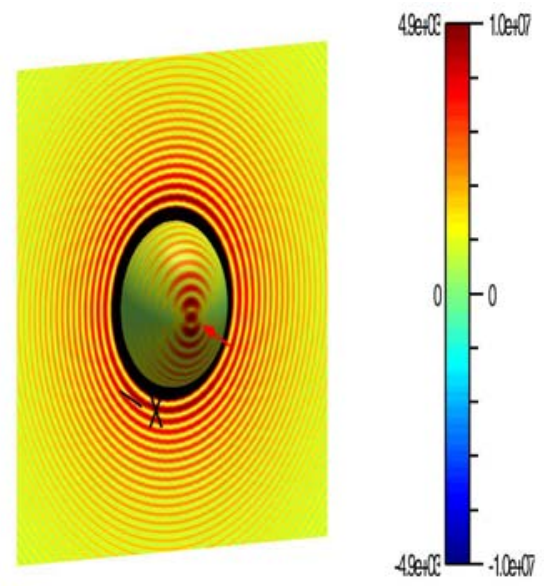

Scattered electric field $|\mathcal{E}(\cdot, t)|$ and surface current $|\boldsymbol{w}(\cdot)|$

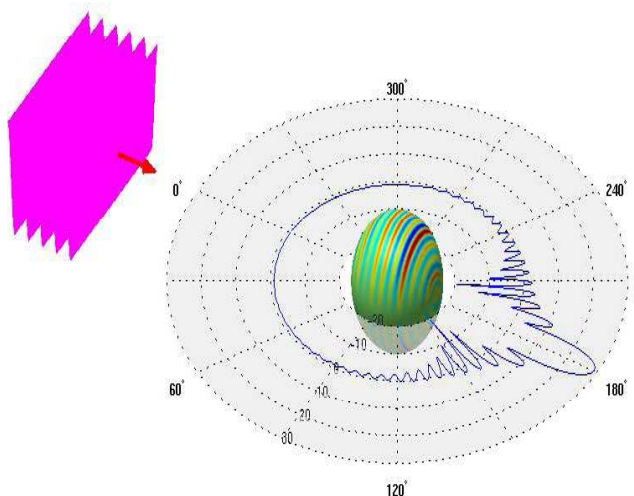

Bistatic RCS

FIG. 4. Visualizations of the scattered field, surface current, and bistatic RCS induced by a horizontally polarized plane wave impinging on $\mathbf{s p h}(10 \lambda)$ at $3.0 \mathrm{GHz}$ with $\widehat{\boldsymbol{d}}_{0}=(-1,0,0)^{T}$.


Scattered electric field $|\mathcal{E}(\cdot, t)|$ and surface current $|\boldsymbol{w}(\cdot)|$

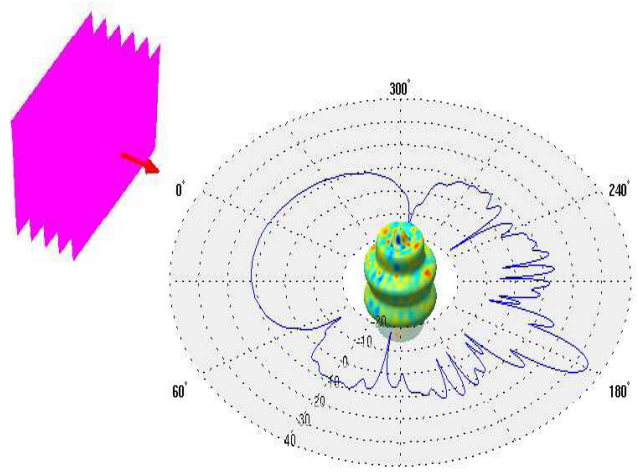

Bistatic RCS

FIG. 5. Visualizations of the scattered field, surface current, and bistatic RCS induced by a horizontally polarized plane wave impinging on hive $(10 \lambda)$ at $1.4 \mathrm{GHz}$ with $\widehat{\boldsymbol{d}}_{0}=(-1,0,0)^{T}$. 

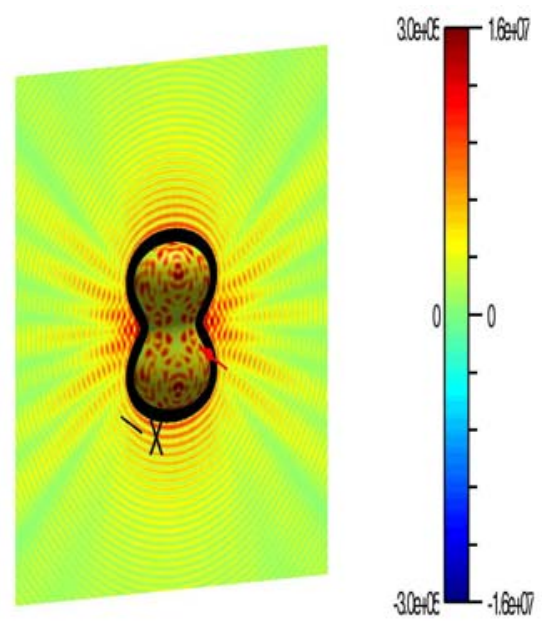

Scattered electric field $|\mathcal{E}(\cdot, t)|$ and surface current $|\boldsymbol{w}(\cdot)|$

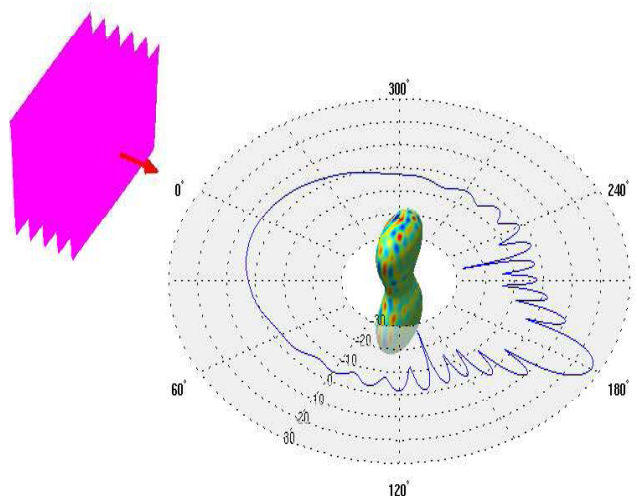

Bistatic RCS

FIG. 6. Visualizations of the scattered field, surface current, and bistatic RCS induced by a horizontally polarized plane wave impinging on $\operatorname{cas}(10 \lambda)$ at $3.0 \mathrm{GHz}$ with $\widehat{\boldsymbol{d}}_{0}=(-1,0,0)^{T}$.
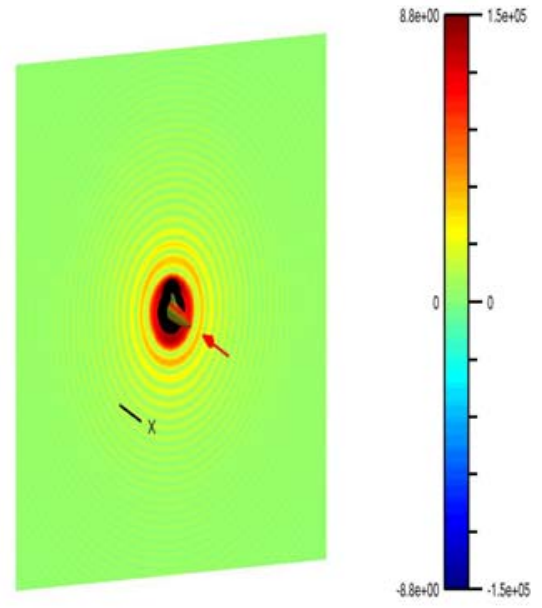

Scattered electric field $|\mathcal{E}(\cdot, t)|$ and surface current $|\boldsymbol{w}(\cdot)|$



Bistatic RCS

FIG. 7. Visualizations of the scattered field, surface current, and bistatic RCS induced by a horizontally polarized plane wave impinging on $\operatorname{sub}(10 \lambda)$ at $149.9 \mathrm{MHz}$ with $\widehat{\boldsymbol{d}}_{0}=(-1,0,0)^{T}$. 



Scattered electric field $|\mathcal{E}(\cdot, t)|$ and surface current $|\boldsymbol{w}(\cdot)|$

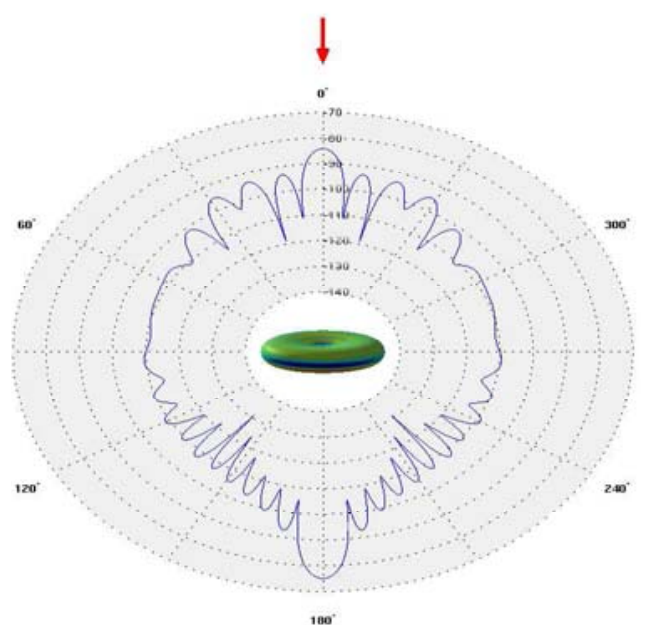

Bistatic RCS

FIG. 8. Visualizations of the scattered field, surface current, and bistatic RCS induced by a horizontally polarized plane wave impinging on ery $(10 \lambda)$ at $475.9 \mathrm{THz}$ with $\widehat{\boldsymbol{d}}_{0}=(0,0,-1)^{T}$.

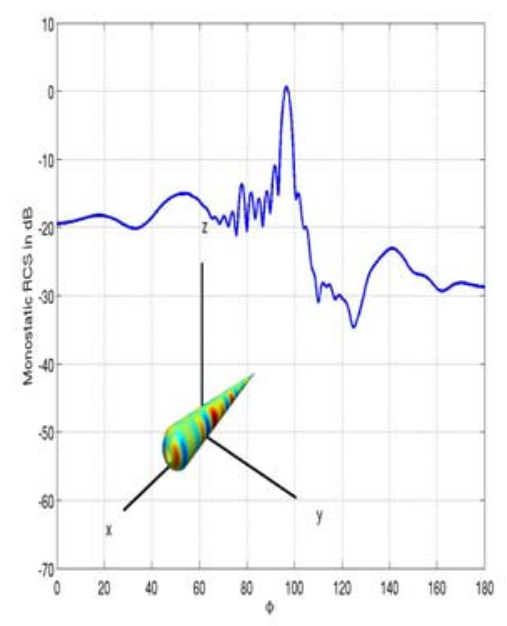

Monostatic RCS and surface current

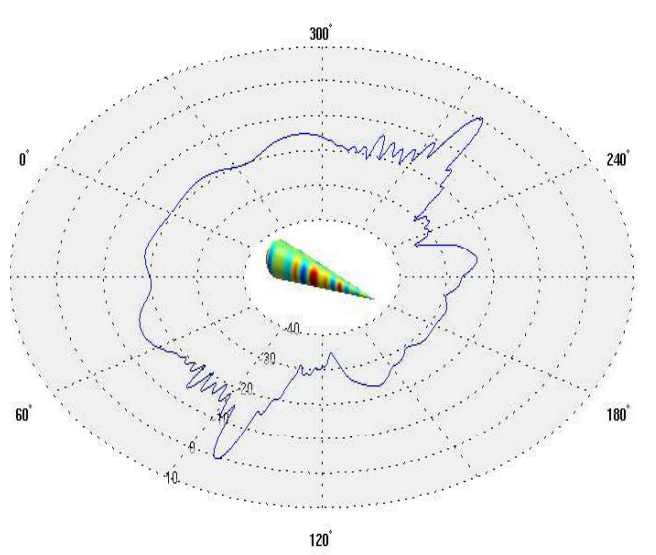

Monostatic RCS

FIG. 9. Visualizations of the surface current and monostatic RCS induced by a vertically polarized plane wave impinging on $\mathbf{c o n e} \mathbf{s p h}_{(10 \lambda)}$ at $4.35 \mathrm{GHz}$. 
[1] G. E. Antilla And N. G. Alexopoulos, Scattering from complex three-dimensional geometries by a curvilinear hybrid finite-element-integral equation approach, J. Opt. Soc. Amer. A, 11 (1994), pp. 1445-1457.

[2] A. Bendali, Y. Boubendir, and M. Fares, A FETI-like domain decomposition method for coupling finite elements and boundary elements in large-size scattering problems of acoustic scattering, Comput. \& Structures, 85 (2007), pp. 526-535.

[3] O. P. Bruno, Fast, high-order, high-frequency integral methods for computational acoustics and electromagnetics, in Topics in Computational Wave Propagation, M. Ainsworth et al., eds., Lect. Notes Comput. Sci. Eng. 31, Springer, Berlin, 2003, pp. 43-82.

[4] O. P. Bruno and L. A. Kunyansky, A fast, high-order algorithm for the solution of surface scattering problems: Basic implementation, tests, and applications, J. Comput. Phys., 169 (2001), pp. 80-110.

[5] L. Canino, J. Ottusch, M. Stalzer, J. Visher, and S. Wandzura, Numerical solution of the Helmholtz equation in $2 D$ and $3 D$ using a high-order Nystrom discretization, J. Comput. Phys., 146 (1998), pp. 627-663.

[6] W. C. Chew, J. M. Jin, E. Michielssen, And J. Song, Fast and Efficient Algorithms in Computational Electromagnetics, Artech House, Norwood, MA, 2001.

[7] D. Colton And R. Kress, Integral Equation Methods in Scattering Theory, Wiley, New York, 1983.

[8] D. Colton And R. Kress, Inverse Acoustic and Electromagnetic Scattering Theory, Springer, Berlin, 1998.

[9] E. Darve And P. Havé, Efficient fast multipole method for low-frequency scattering, J. Comput. Phys., 197 (2004), pp. 341-363.

[10] E. Darve AND P. HaVÉ, A fast multipole method for Maxwell equations stable at all frequencies, Phil. Trans. Roy. Soc. London Ser. A, 362 (2004), pp. 603-628.

[11] W. Freeden, T. Gervens, and M. Schreiner, Constructive Approximation on the Sphere, Oxford University Press, Oxford, UK, 1998

[12] M. Ganesh And I. G. GRAham, A high-order algorithm for obstacle scattering in three dimensions, J. Comput. Phys., 198 (2004), pp. 211-242.

[13] M. Ganesh, I. G. Graham, and J. Sivaloganathan, A pseudospectral three-dimensional boundary integral method applied to a nonlinear model problem from finite elasticity, SIAM J. Numer. Anal., 31 (1994), pp. 1378-1414.

[14] M. Ganesh, I. G. Graham, and J. Sivaloganathan, A new spectral boundary integral collocation method for three-dimensional potential problems, SIAM J. Numer. Anal., 35 (1998), pp. $778-805$.

[15] M. Ganesh and S. C. Hawkins, An efficient surface integral equation method for the timeharmonic Maxwell equations, ANZIAM J., 48 (2007), pp. C17-C33.

[16] M. Ganesh AND S. C. HAWkins, A spectrally accurate algorithm for electromagnetic scattering in three dimensions, Numer. Algorithms, 43 (2006), pp. 25-60.

[17] I. G. GRaham AND I. H. Sloan, Fully discrete spectral boundary integral methods for Helmholtz problems on smooth closed surfaces in $\mathbb{R}^{3}$, Numer. Math., 92 (2002), pp. 289-323.

[18] E. Heikkola, T. Rossi, and J. Toivanen, A parallel fictitious domain method for the threedimensional Helmholtz equation, SIAM J. Sci. Comput., 24 (2003), pp. 1567-1588.

[19] J. Hellmers, E. Eremina, And T. Wriedt, Simulation of light scattering by biconcave Cassini ovals using the nullfield method with discrete sources, J. Opt. A: Pure Appl. Opt., 8 (2006), pp. 1-9.

[20] E. F. Knott, J. F. Shaeffer, and M. T. Tuley, Radar Cross Section, SciTech Publishing, Raleigh, NC, 2004.

[21] P. Monk, Finite Element Methods for Maxwell's Equations, Oxford University Press, Oxford, UK, 2003.

[22] R. ReIMER, Hyperinterpolation on the sphere at the minimal projection order, J. Approx. Theory, 103 (2000), pp. 272-286.

[23] F. Reitich, K. K. Tamma, and R. R. Namburu, eds., Special Issue on Computational Electromagnetics, Comput. Model. Eng. Sci., 5 (2004).

[24] I. H. Sloan And R. S. Womersley, Constructive polynomial approximation on the sphere, J. Approx. Theory, 103 (2000), pp. 91-118.

[25] J. M. Song, C. C. Lu, W. C. Chew, And S. W. Lee, Fast Illinois solver code (FISC), IEEE Antennas Propag. Mag., 40 (1998), pp. 27-34.

[26] R. A. R. Tricker, Introduction to Meteorological Optics, Elsevier, New York, 1970. 
[27] L. Tsang, J. A. Kong, And K. Ding, Scattering of Electromagnetic Waves: Theories and Applications, Wiley, New York, 2000.

[28] R. T. Wang And H. C. van DE Hulst, Rainbows: Mie computations and the Airy approximation, Appl. Optics, 30 (1991), pp. 106-117.

[29] L. Wienert, Die Numerische Approximation von Randintegraloperatoren für die Helmholtzgleichung im $\mathbb{R}^{3}$, Ph.D. thesis, University of Göttingen, Göttingen, Germany, 1990.

[30] A. C. Woo, H. T. Wang, M. J. Schuh, and M. L. Sanders, Benchmark radar targets for the validation of computational electromagnetics programs, IEEE Antennas Propag. Mag., 35 (1993), pp. 84-89.

[31] T. Wriedt, J. Hellmers, E. Eremina, And R. Schuh, Light scattering by single erythrocite: Comparison of different methods, J. Quant. Spectrosc. Radiat. Transfer, 100 (2006), pp. 444-456. 